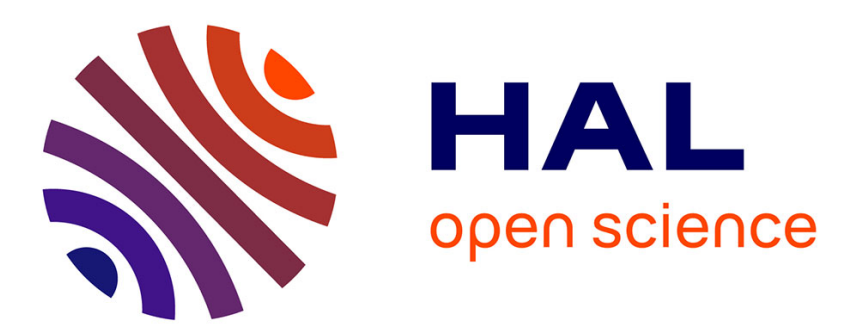

\title{
A micromechanics-based model for shear-coupled grain boundary migration in bicrystals
}

\author{
Stephane Berbenni, Bhasker Paliwal, Mohammed Cherkaoui
}

\section{To cite this version:}

Stephane Berbenni, Bhasker Paliwal, Mohammed Cherkaoui. A micromechanics-based model for shear-coupled grain boundary migration in bicrystals. International Journal of Plasticity, 2013, 44, pp.68-94. 10.1016/j.ijplas.2012.11.011 . hal-01947296

\section{HAL Id: hal-01947296 \\ https://hal.science/hal-01947296}

Submitted on 6 Dec 2018

HAL is a multi-disciplinary open access archive for the deposit and dissemination of scientific research documents, whether they are published or not. The documents may come from teaching and research institutions in France or abroad, or from public or private research centers.
L'archive ouverte pluridisciplinaire HAL, est destinée au dépôt et à la diffusion de documents scientifiques de niveau recherche, publiés ou non, émanant des établissements d'enseignement et de recherche français ou étrangers, des laboratoires publics ou privés. 


\title{
A micromechanics-based model for shear-coupled grain boundary migration in bicrystals
}

\author{
Stéphane Berbenni ${ }^{\mathrm{a}, \mathrm{b}, 1}$, Bhasker Paliwal ${ }^{\mathrm{b}, \mathrm{c}}$, \\ Mohammed Cherkaoui b,c \\ a Laboratoire d'Etude des Microstructures et de Mécanique des Matériaux, LEM3, \\ UMR CNRS 7239, University of Lorraine, Ile du Saulcy, 57045 Metz, France \\ ${ }^{\mathrm{b}}$ Unité Mixte Internationale Georgia Tech Lorraine-CNRS, UMI CNRS 2958, \\ Technopole, 57070 Metz, France \\ ${ }^{\mathrm{c}}$ George W. Woodruff School of Mechanical Engineering, Georgia Institute of \\ Technology, Atlanta, GA 30332-0405, USA
}

\begin{abstract}
A complete micromechanics-based model is here proposed using the concepts of continuum kinematics and thermodynamics. A new constitutive framework is proposed to describe stress-induced "shear-coupled" grain boundary (GB) migration. Like non diffusive phase-transformations, shear-coupled GB migration can be considered on the thermodynamics point of view of conservative nature until high temperature with respect to melting point (i.e. diffusionless but thermally activated). The micromechanics-based continuum model can include intra-crystalline slip, GB sliding and shear-coupled GB migration as additive dissipative mechanisms. To illustrate the present theory, the model is applied to shear-coupled GB
\end{abstract}


migration in the case of three "flat" $\mathrm{Cu}$ bi-crystals [001] with symmetric tilt GB (STGB): $\Sigma 17(410)\left(\theta=28.07^{\circ}\right), \Sigma 5(210)\left(\theta=53.13^{\circ}\right), \Sigma 41(540)\left(\theta=77.32^{\circ}\right)$. Molecular dynamics (MD) simulations under simple shear loading are first performed to identify the active shear coupling modes, the stick-slip behaviour at $0 \mathrm{~K}$ and $500 \mathrm{~K}$ and the bicrystal finite size dependence on the shear stress responses. The results of the micromechanical model are discussed in comparison with MD simulations. The effects of anisotropic vs. isotropic elastic properties on effective elastic shear moduli, overall shear stress drop magnitudes and dissipated energy during GB migration are analyzed for these STGB.

Keywords: Grain boundary motion; Constitutive behaviour; Micromechanics; Molecular dynamics; Copper

\section{Introduction}

Interfacial or grain boundary (GB) constitutive behaviour that takes into account possible GB migration and grain size/shape changes at different temperatures is today seen relevant to understand the complex mechanisms in grain boundary engineering and nanocrystalline (NC) materials (Sutton and Balluffi, 1995; Cahn and Taylor, 2004; Cahn et al., 2006a,b; Gianola et al., 2006, 2008; Mompiou et al., 2009, 2010, 2011; Farkas et al., 2006; Tucker et al., 2010, 2011; Tucker and McDowell, 2011). Furthermore, bridging methods between atomistic phenomena at GB and continuum mechanics should be developed for the design of new engineering materials that involve multiple plasticity mechanisms and various length scales (McDowell, $2008,2010)$. The inelastic deformation accommodation mechanisms associated to GB

$\overline{1}$ Corresponding author.

Email address: Stephane.Berbenni@univ-lorraine.fr (Stéphane Berbenni) 
significantly affect the mechanical behaviour of polycrystals.

Among these mechanisms, "shear-coupled" GB migration is now seen to compete or interplay with other intra-granular GB mechanisms in a wide range of temperatures (Cahn et al., 2006a,b). In NC metals, it now becomes challenging to understand stress-induced GB migration because this is thought to enhance grain growth at low temperatures, which is important for making stable structural materials for engineering applications (Jin et al., 2004; Zhang et al., 2005). In these materials, the interplay of GB migration with other possible GB deformation mechanisms like GB sliding (Warner et al., 2006), grain rotation, diffusional creep, partial or perfect dislocation nucleation and absorption at GB (Bobylev et al., 2010) becomes very complex due to the high GB volume fraction. For instance, GB migration and pure sliding can be considered as additive mechanisms which may both be present at high temperature (Yoshida et al., 2002). In real polycrystalline metals, GB mobility is also very difficult to capture because of different GB characters (Gottstein and Shvindlerman, 2010).

Stress-induced GB migration was first discovered experimentally in the 1950's (Li et al., 1953) for low-angle grain boundaries (LAGB). For LAGB, stress-induced GB migration was interpreted in terms of collective motion of single dislocations (Read and Shockley, 1950; Read, 1953). In addition, high-angle GB (HAGB) were also seen to move under applied stress (Biscondi and Goux, 1968). More recently, many experimental data (Winning et al., 2001, 2002; Molodov et al., 2007; Gorkaya et al., 2009; Molodov et al., 2011) confirmed this stress-induced GB mechanism for highpurity planar Al bi-crystals with both [001] tilt LAGB and HAGB (from 0 to $90^{\circ}$ misorientations). By performing mechanical tests on $\mathrm{Al}$ bi-crystals on a wide range of temperature (from $280^{\circ} \mathrm{C}$ to $400^{\circ} \mathrm{C}$ ), Gorkaya et al. (2009) showed that GB migration is a thermally activated process and there is a misorientation dependence of 
activation parameters like activation enthalpy and mobility pre-exponential factor. The mechanism of stress-induced shear-coupled GB migration at room temperature is today well identified by a shear deformation accompanying GB migration for symmetric (coincident) tilt GB (here denoted STGB) but less for general non symmetric GB. This new deformation mechanism is different from strain-induced GB migration studied for recrystallization phenomena. The latter essentially comes from spatially heterogeneous intra-crystalline dislocation densities in the vicinity of GB (Busso, 1998).

In the same time, theoretical studies (Read and Shockley, 1950; Read, 1953; Cahn and Taylor, 2004) as well as molecular dynamics (MD) simulations using the EAM potential for Cu bicrystals with STGB (Cahn et al., 2006a,b; Zhang et al., 2008) show that stress-induced GB migration is characterized by a shear "coupling factor" (or shear deformation usually denoted $\beta$ ) which is defined by the ratio of the shear displacement parallel to the GB plane to the GB propagation normal to its plane. This coupling factor is purely geometric and depends on the tilt GB misorientation. "Shear-coupled" GB migration was recently analyzed by Cahn et al. (2006a,b) using the "Frank-Bilby" equation (Frank, 1950; Bilby, 1955; Bullough and Bilby, 1956).

By performing in situ TEM observations of different grains with curved GB in stressed nano-polycrystalline Al films, Legros et al. (2008) found that GB motion essentially occurs near stress concentration regions like cracks with very large velocities $\left(30 \mathrm{~nm} . \mathrm{s}^{-1}-200 \mathrm{~nm} \cdot \mathrm{s}^{-1}\right)$. This would indicate that stress-induced GB migration is a new relaxation mechanism different from long-range diffusion processes at GB. For that reason, it is very similar to stress induced twin boundary migration, which was very recently finely examined through MD simulations (Tucker et al., 2011; Wang et al., 2011). Mompiou et al. (2009) also experimentally confirmed in ultrafine grained $\mathrm{Al}$ with random $\mathrm{GB}$ the minor role of diffusion in ultra-fine grained $\mathrm{Al}$ 
with random GB during the GB migration process and the absence of GB dislocation emission. They showed a coupling factor exists between shear and migration, and their measurement for HAGB was close to the theoretical value derived by Cahn et al. (2006a,b). Later, the same group (Mompiou et al., 2010, 2011) developed a purely geometric approach, called "SMIG model", for GB not having necessarily specific orientation relationships. For general GB, shear-coupled GB migration involves the glide motion of more generalized defects than dislocations called "disconnections" (Hirth and Pond, 1996; Hirth et al., 2007; Pond et al., 2008).

Due to the complexity of atomistic mechanisms in the case of general GB, we will limit the present study to the constitutive behaviour of Cu STGB undergoing shearcoupled migration. For $\mathrm{Cu}$ [001] STGB, two shear deformation modes associated to $<110>$ and $<100>$ crystallographic directions linked to two coupling factors (resp. negative and positive) were observed using MD simulations and confirmed experimentally by Molodov et al. (2007, 2011). In particular, a dual temperature dependent behaviour for certain misorientations (around $53^{\circ}$ ) may be observed at finite temperatures. According to Cahn et al. (2006a), a transition exists above 800K where the shear-coupled GB migration may be interrupted by occasional sliding events. Between these sliding events, the GB plane continues to move accompanied by shear. This suggests that pure GB sliding occurs through atomistic mechanisms that preserve GB character. At medium and low temperatures, shear-coupled GB migration has a stick slip stress versus time characteristic response which can be retrieved by atomistic simulations (Mishin et al., 2007; Ivanov and Mishin, 2008).

The objectives of the present paper are the following. A complete micromechanicsbased constitutive model is here proposed using the concepts of continuum thermomechanics to describe shear-coupled GB migration in bicrystals. Kinematics and thermodynamics associated with different additive dissipative mechanisms (intra- 
crystalline slip, GB sliding and shear-coupled GB migration) will be introduced in section 2. In this paper, shear-coupled GB migration will be considered as a shear process in the local coordinates associated to grain boundary plane that can be described in linearized kinematics by an eigenstrain (or plastic strain jump at the discontinuity GB interface) similarly to deformation twinning (Christian and Mahajan, 1995; Fischer et al., 2003). To illustrate the present theory, section 3 will focus on planar bicrystals and pure shear-coupled situations without sliding in addition to intra-crystalline slip. It will be derived that the shear "coupling factor" $\beta$ is related to plastic strain jump at the GB through an "orientation tensor" characterized by GB interfacial Burgers vector and slip plane. In section 4, numerical examples and MD simulations will be restricted to the shear responses of three $\mathrm{Cu}$ STGB exhibiting shear-coupled GB migration with absence of bulk plasticity (crystal sizes will be lower or equal to $10 \mathrm{~nm}$ ). In these situations, atomic scale deformation mechanisms are well identified using the concept of "displacement shift complete" (DSC) dislocations (Rae and Smith, 1980; Sutton and Balluffi, 1995; Cahn et al., 2006a) or "disconnections" (Hirth and Pond, 1996; Hirth et al., 2007; Pond et al., 2008). These interfacial defects will be introduced in the constitutive framework and a discussion about the role of stress-induced GB migration coupled with anisotropic elasticity on stress-strain characteristics is provided in the light of the micromechanics-based model. Section 5 concludes and sketch some perspectives for the applicability of the present bi-crystal constitutive framework in mean field polycrystalline modelling involving NC materials and/or deformation twinning.

Throughout the paper, a "," indicates a spatial differentiation, a superposed dot a particle time derivative (or rate). "[A]" denotes the jump of a bulk field "A" at a discontinuity surface such that $[\mathrm{A}]=\mathrm{A}^{\mathrm{II}}-\mathrm{A}^{\mathrm{I}}$ to be consistent with Fig. 1 where $\mathrm{I}$ and II are both crystals forming a bicrystal (crystal II being the consumed crystal during interface motion). " $\langle\mathrm{A}\rangle$ " denotes the average of a bulk field A across the 
interface defined by $\langle\mathrm{A}\rangle=\frac{1}{2}\left(\mathrm{~A}^{\mathrm{II}}+\mathrm{A}^{\mathrm{I}}\right)$. The Einstein summation convention is also used throughout the paper.

\section{Continuum thermo-micromechanical model}

\subsection{Kinematics}

Following Abeyaratne and Knowles (1990), Cermelli and Gurtin (1994), Simha and Bhattacharya (1998), Fischer et al. (1998), the particle velocity vector jump at the discontinuity interface (GB) denoted hereafter $S$ can be decomposed as follows (Fig. 1)

$$
\left[v_{i}\right]=\left[v_{i}\right]^{(1)}+\left[v_{i}\right]^{(2)}
$$

where

$$
\left[v_{i}\right]^{(1)}=-\left[u_{i, j}\right] n_{j} \omega_{N}
$$

is the particle velocity jump due to normal GB propagation assuming linearized kinematics (Hadamard, 1903; Abeyaratne and Knowles, 1990) and $\left[v_{i}\right]^{(2)}$ is the part of particle velocity jump at the interface due to tangential GB sliding. In Eq. $2,\left[u_{i, j}\right]$ represents the jump of displacement gradient or total distortion at the discontinuity interface $S . \omega_{N}$ is the GB normal velocity and $n_{i}$ is the unit normal vector to the GB plane oriented from I towards II (Fig. 1). According to Fig. 1, the particle velocity jump contains a tangential part $v^{\|}$and a normal one $v^{\perp}$ as follows

$$
\left[v_{i}\right]=v^{\|} t_{i}+v^{\perp} n_{i}
$$

where $v^{\|}$reads

$$
v^{\|}=\beta \omega_{N}+v_{s}
$$

In Eq. $4, v_{s}=\left[v_{i}\right]^{(2)} t_{i}$ is the tangential velocity due to GB sliding. This one has to be specified by a constitutive law depending on a driving force. The constitutive 
law for tangential GB sliding is not considered because only pure shear-coupled GB migration configurations will be presented in the rest of the paper from Section 3. Using Eq. 2, $\beta$ can be identified as a function of the interfacial jump of displacement gradient through the following expression

$$
\beta=-\left[u_{i, j}\right] n_{j} t_{i}
$$

$\beta$ is a purely geometric parameter due to interfacial kinematics that can be identified as the "coupling factor" following the terminology used by Cahn and Taylor (2004). This section shows it does not depend on any constitutive law.

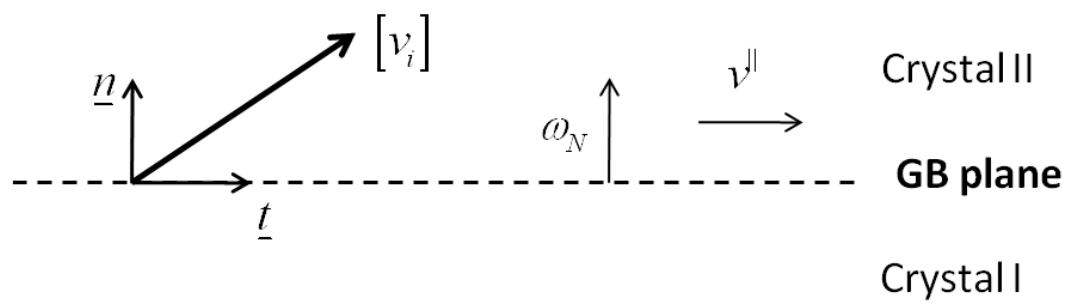

Figure 1. Schematic representation of grain boundary (GB) migration and sliding (kinematics). $\left[v_{i}\right]=v_{i}{ }^{I I}-v_{i}^{I}$ denotes the jump of $v_{i}$ at the interface (GB plane) oriented by unit normal vector $n_{i}$ from crystal I to crystal II.

In the case of a perfect interface (without GB sliding), the jump of displacement gradient reads

$$
\left[u_{i, j}\right]=\lambda_{i} n_{j}
$$

where $\lambda_{i}$ is an arbitrary vector measuring the magnitude and direction of displacement gradient jump at the interface (this one can be obtained from the so-called Christoffel matrix). It is noteworthy that Eq. 4 was postulated by Cahn and Taylor (2004) without any direct link to continuum-based kinematics like in the present contribution. Eq. 5 may serve to link the "coupling factor" $\beta$ to the shear deformation component as revealed by Tucker et al. (2011) using atomistic simulations and associated deformation metrics. Their Fig. $4 c$ shows a typical shear-coupled GB migration process for a $\Sigma 3(111)$ coherent twin boundary in f.c.c. materials. According 
to Eq. 5, this leads to a "coupling factor" close to $1 / \sqrt{2}$ which is consistent with twinning shear deformation in f.c.c. materials (Christian and Mahajan, 1995; Fischer et al., 2003).

\subsection{Thermodynamics}

The mechanical dissipation $D$ in the body $V$ is defined as the difference between the power of the applied forces denoted $P_{\text {ext }}$ and the rate of change of the stored energy $\dot{\Phi}$ (time derivative of the Helmholtz free energy), which corresponds under isothermal and quasi-static evolutions to the time derivative of the elastic energy (Coleman and Gurtin, 1967; Maugin, 1992; Maugin and Muschik, 1994; Maugin, 2011)

$$
D=P_{e x t}-\dot{\Phi}
$$

Neglecting the excess interfacial energy effects at GB in Eq. 7, $\Phi$ is given by

$$
\Phi=\int_{V} \frac{1}{2} \sigma_{i j} \varepsilon_{i j}^{e} d V
$$

where $\sigma_{i j}$ and $\varepsilon_{i j}^{e}$ are respectively the Cauchy stresses and the elastic strains. The power of external forces is defined as

$$
P_{e x t}=\int_{\partial V} \sigma_{i j} n_{j} v_{i} d V
$$

where $n_{j}$ is the unit outward normal vector at a point of the external boundary of $V$ denoted $\partial V$ and $v_{i}$ is the material velocity at this point. As described in the kinematics part (Subsection 2.1), the strains and stresses are discontinuous across the moving interface. Consequently, the elastic energy density $\phi=\frac{1}{2} \sigma_{i j} \varepsilon_{i j}^{e}$ present in Eq. 8 is also discontinuous through the moving interface $S$. Applying the transport theorem for growing discontinuity interface $S$ to Eq. 8, $\dot{\Phi}$ is given by (see also 
Cherkaoui et al. (1998, 2000); Langlois and Berveiller (2003))

$$
\dot{\Phi}=\int_{V} \dot{\phi} d V-\int_{S}[\phi] \omega_{N} d S
$$

In Eq. 10, the first volume term containing $\dot{\phi}$ can be easily computed using $\varepsilon_{i j}=$ $\varepsilon_{i j}^{e}+\varepsilon_{i j}^{p}$ as follows

$$
\int_{V} \dot{\phi} d V=\int_{V} \sigma_{i j}\left(\dot{\varepsilon}_{i j}-\dot{\varepsilon}_{i j}^{p}\right) d V
$$

The second term of Eq. 10 which contains $[\phi]$ is defined as

$$
[\phi]=\frac{1}{2}\left(\sigma_{i j}^{I I}\left(\varepsilon_{i j}^{I I}-\varepsilon_{i j}^{p I I}\right)-\sigma_{i j}^{I}\left(\varepsilon_{i j}^{I}-\varepsilon_{i j}^{p I}\right)\right)
$$

This expression is much simplified in the case of linear homogeneous elastic properties and using the usual symmetries of the homogeneous elastic stiffness tensor $C_{i j k l}$ as follows

$$
[\phi]=\left\langle\sigma_{i j}\right\rangle\left[\varepsilon_{i j}-\varepsilon_{i j}^{p}\right]
$$

Thus, the expression for $\dot{\Phi}$ is obtained using Eqs. 10-12

$$
\dot{\Phi}=\int_{V} \sigma_{i j}\left(\dot{\varepsilon}_{i j}-\dot{\varepsilon}_{i j}^{p}\right) d V-\int_{S}[\phi] \omega_{N} d S
$$

The expression of external power $P_{\text {ext }}$ can be simplified using the divergence theorem starting from Eq. 9 as follows

$$
\begin{aligned}
& P_{\text {ext }}=\int_{V}\left(\sigma_{i j} v_{i}\right)_{, j} d V+\int_{S}\left[\sigma_{i j} v_{i}\right] n_{j} d V= \\
& \int_{V}\left(\sigma_{i j, j} v_{i}+\sigma_{i j} \dot{\varepsilon}_{i j}\right) d V+\int_{S}\left(\left\langle\sigma_{i j} n_{j}\right\rangle\left[v_{i}\right]+\left[\sigma_{i j} n_{j}\right]\left\langle v_{i}\right\rangle\right) d S
\end{aligned}
$$

Because of the identity $\left[\sigma_{i j} v_{i}\right]=\left\langle\sigma_{i j}\right\rangle\left[v_{i}\right]+\left[\sigma_{i j}\right]\left\langle v_{i}\right\rangle$ (Abeyaratne and Knowles, 1990), static stress equilibrium without body forces in the bulk $\left(\sigma_{i j, j}=0\right)$ and traction vector continuity across $S$ (i.e. $\left[\sigma_{i j} n_{j}\right]=0$ ) applied to Eq. 15 yields

$$
P_{e x t}=\int_{V} \sigma_{i j} \dot{\varepsilon}_{i j} d V+\int_{S}\left\langle\sigma_{i j} n_{j}\right\rangle\left[v_{i}\right] d S
$$


Introducing Eqs. 1 and 2 in Eq. 16, it comes

$$
P_{e x t}=\int_{V} \sigma_{i j} \dot{\varepsilon}_{i j} d V-\int_{S}\left\langle\sigma_{i j} n_{j}\right\rangle\left[u_{i, k}\right] n_{k} \omega_{N} d S+\int_{S}\left\langle\sigma_{i j} n_{j}\right\rangle\left[v_{i}\right]^{(2)} d S
$$

From Eq. 6, the following identity holds $\left\langle\sigma_{i j} n_{j}\right\rangle\left[u_{i, k}\right] n_{k}=\left\langle\sigma_{i j} n_{j}\right\rangle \lambda_{i} n_{k} n_{k}=\left\langle\sigma_{i j}\right\rangle \lambda_{i} n_{j}=$ $\left\langle\sigma_{i j}\right\rangle\left[u_{i, j}\right]=\left\langle\sigma_{i j}\right\rangle\left[\varepsilon_{i j}\right]$. Thus, Eq. 17 simplifies into

$$
P_{e x t}=\int_{V} \sigma_{i j} \dot{\varepsilon}_{i j} d V-\int_{S}\left\langle\sigma_{i j}\right\rangle\left[\varepsilon_{i j}\right] \omega_{N} d S+\int_{S}\left\langle\sigma_{i j} n_{j}\right\rangle\left[v_{i}\right]^{(2)} d S
$$

By comparing Eq. 14 and Eq. 18, the total dissipation $D$ of the system is positive and reads according to Eq. 7

$$
D=\int_{V} \sigma_{i j} \dot{\varepsilon}_{i j}^{p} d V-\int_{S}\left(\left\langle\sigma_{i j}\right\rangle\left[\varepsilon_{i j}\right]-[\phi]\right) \omega_{N} d S+\int_{S}\left\langle\sigma_{i j} n_{j}\right\rangle\left[v_{i}\right]^{(2)} d S
$$

For homogeneous elastic properties, Eq. 13 can be applied so that Eq. 19 simplifies into

$$
D=\int_{V} \sigma_{i j} \dot{\varepsilon}_{i j}^{p} d V-\int_{S}\left\langle\sigma_{i j}\right\rangle\left[\varepsilon_{i j}^{p}\right] \omega_{N} d S+\int_{S}\left\langle\sigma_{i j} n_{j}\right\rangle\left[v_{i}\right]^{(2)} d S
$$

The first term in Eq. 19 is the classic bulk dissipation due to crystallographic slip evolution in crystals without discontinuity interface. The second term in Eq. 19 is due to the propagation of interface discontinuities and can be related to shearcoupled GB migration. The associated driving force on the interface $S$ is given by $[\phi]-\left\langle\sigma_{i j}\right\rangle\left[\varepsilon_{i j}\right]$ for heterogeneous elastic solids and simplifies into $-\left\langle\sigma_{i j}\right\rangle\left[\varepsilon_{i j}^{p}\right]$ for homogeneous elastic ones. This driving force can be related to the energy-momentum tensor $P_{l j}=\phi \delta_{l j}-\sigma_{i j} u_{i, l}$ introduced by Eshelby $(1951,1970)$ through the jump relationship $\left[P_{l j}\right] n_{j}=\left([\phi]-\left\langle\sigma_{i j}\right\rangle\left[\varepsilon_{i j}\right]\right) n_{l}$ for heterogeneous elastic solids. The last term in Eq. 19 or Eq. 20 is due to a possible incoherent interface authorizing tangential GB sliding (see Eq. 4.). In the following, we first highlight the application of the continuum kinematics and thermodynamics frameworks to stress-induced shear-coupled GB migration. From the continuum mechanics viewpoint, GB is here considered as a continuously distributed dislocation (in the sense of collective dislocations) for both 
LAGB and HAGB. In section 3, the transport of GB dislocations is fully examined considering a bicrystal with planar GB and average mechanical fields in each crystal.

\section{Bicrystals with planar grain boundaries and perfect shear-coupled mi- gration}

\subsection{Transport equations and average fields}

In this part and in the rest of the paper, interfacial sliding will be disregarded, and we only focus on stress-induced motion of discontinuity interfaces like GB, assuming they are coherent interfaces. This means that only the first two terms of Eq. 19 are considered. This situation corresponds to "perfect shear-coupling" GB migration as defined in Cahn and Taylor (2004). Thus, for a bicrystals with planar GB, such as the one represented in Fig. 2, Eq. 19 yields the dissipation per unit volume

$$
\frac{D}{V}=f \sigma_{i j}^{I} \dot{\varepsilon}_{i j}^{p I}+(1-f) \sigma_{i j}^{I I} \dot{\varepsilon}_{i j}^{p I I}+\left([\phi]-\left\langle\sigma_{i j}\right\rangle\left[\varepsilon_{i j}\right]\right) \dot{f}
$$

where $f$ is the current volume fraction of crystal I. If crystal I moves into crystal II, $\dot{f}$ describes the rate of growth of the thickness of crystal I due to normal motion. In the particular case of homogeneous elasticity, $[\phi]-\left\langle\sigma_{i j}\right\rangle\left[\varepsilon_{i j}\right]$ should be replaced by $-\left\langle\sigma_{i j}\right\rangle\left[\varepsilon_{i j}^{p}\right]$ in Eq. 21. Furthermore, the overall strain (resp. stress) evolutions are given by the following transport equations (see also Petryk (1998)) involving the strain (resp. stress) jump $\left[\varepsilon_{i j}\right]$ (resp. $\left[\sigma_{i j}\right]$ ) for the plane discontinuity interface $S$

$$
\begin{aligned}
& \dot{E}_{i j}=\frac{1}{V} \int_{V} \dot{\varepsilon}_{i j} d V-\frac{1}{V} \int_{S}\left[\varepsilon_{i j}\right] \omega_{N} d S=f \dot{\varepsilon}_{i j}^{I}+(1-f) \dot{\varepsilon}_{i j}^{I I}-\left[\varepsilon_{i j}\right] \dot{f} \\
& \dot{\Sigma}_{i j}=\frac{1}{V} \int_{V} \dot{\sigma}_{i j} d V-\frac{1}{V} \int_{S}\left[\sigma_{i j}\right] \omega_{N} d S=f \dot{\sigma}_{i j}^{I}+(1-f) \dot{\sigma}_{i j}^{I I}-\left[\sigma_{i j}\right] \dot{f}
\end{aligned}
$$




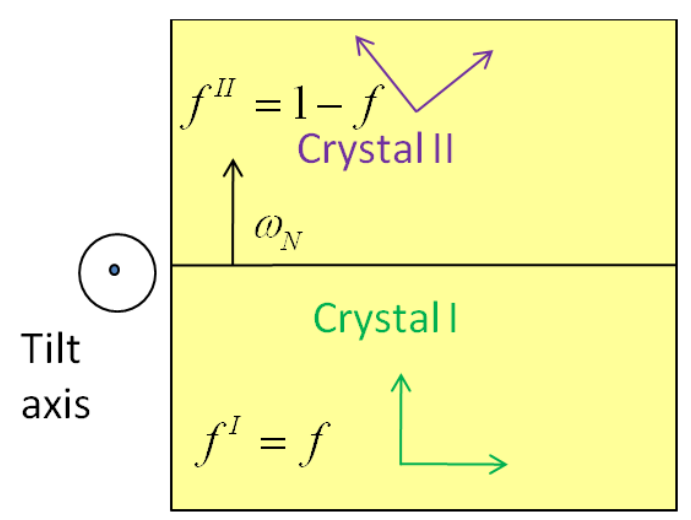

Figure 2. Bicrystal configuration for shear-coupled GB migration with infinite planar interface (GB plane). Here, the consumed grain (crystal II) is chosen as the reference lattice. From Eq. 22 and assuming homogeneous elasticity, the overall plastic strain rate reads

$$
\dot{E}_{i j}^{p}=\frac{1}{V} \int_{V} \dot{\varepsilon}_{i j}^{p} d V-\frac{1}{V} \int_{S}\left[\varepsilon_{i j}^{p}\right] \omega_{N} d S=f \dot{\varepsilon}_{i j}^{p I}+(1-f) \dot{\varepsilon}_{i j}^{p I I}-\left[\varepsilon_{i j}^{p}\right] \dot{f}
$$

For an infinite plane GB and considering heterogeneous elasticity, the strain concentration equations (given applied homogeneous strains) are detailed in Appendix A through Eq. A.3. The calculations give the following general expressions for $\varepsilon_{i j}^{I}$ and $\varepsilon_{i j}^{I I}$

$$
\begin{aligned}
& \varepsilon_{i j}^{I}=A_{i j k l}^{I} E_{k l}-(1-f) G_{i j k l}\left[\sigma_{k l}^{p}\right] \\
& \varepsilon_{i j}^{I I}=A_{i j k l}^{I I} E_{k l}+f G_{i j k l}\left[\sigma_{k l}^{p}\right]
\end{aligned}
$$

where $E_{i j}$ are the overall homogeneous strains, $A_{i j k l}^{I}, A_{i j k l}^{I I}$ are respectively the strain concentration tensors for crystals I and II, $G_{i j k l}$ is a strain influence tensor which depends on the anisotropic elastic constants in crystals I and II. In Eq. 25, $\left[\sigma_{i j}^{p}\right]$ is defined by

$$
\left[\sigma_{i j}^{p}\right]=C_{i j k l}^{I I} \varepsilon_{k l}^{p I I}-C_{i j k l}^{I} \varepsilon_{k l}^{p I}
$$

$A_{i j k l}^{I}, A_{i j k l}^{I I},\left[\sigma_{i j}^{p}\right]$ and $G_{i j k l}$ are given in Appendix A through Eqs. A.4 and A.5. The strain jump $\left[\varepsilon_{i j}\right]$ can be easily derived from Eq. 25. In addition, the effective (overall) elastic moduli $C_{i j k l}^{e f f}$ of the bicrystal can be computed using Eq. 25 together 
with $\left[\sigma_{i j}^{p}\right]=0$ and the static averaging rules. The complete expressions of the effective elastic moduli $C_{i j k l}^{e f f}$ are given in Appendix A through Eqs. A.6 and Eq. A.7. Conversely, the effective (overall) elastic compliances are also given in Appendix A (Eqs. A.11 and A.12). For an infinite planar GB, and assuming heterogeneous elasticity, the analytical expressions (the so-called stress concentration equations) for $\sigma_{i j}^{I}$ and $\sigma_{i j}^{I I}$ are derived in Appendix A through Eq. A.8 and take the general form of

$$
\begin{aligned}
& \sigma_{i j}^{I}=B_{i j k l}^{I} \Sigma_{k l}+(1-f) F_{i j k l}\left[\varepsilon_{k l}^{p}\right] \\
& \sigma_{i j}^{I I}=B_{i j k l}^{I I} \Sigma_{k l}-f F_{i j k l}\left[\varepsilon_{k l}^{p}\right]
\end{aligned}
$$

where $\Sigma_{i j}$ are the overall stresses, $B_{i j k l}^{I}, B_{i j k l}^{I I}$ are respectively the stress concentration tensors for crystals I and II, $F_{i j k l}$ is a stress influence tensor which depends on the anisotropic elastic constants in crystals I and II (see also the works of Stupkiewicz and Petryk (2002); Franciosi and Berbenni (2007, 2008); Richeton and Berbenni (2013)). The complete analytical expressions for $B_{i j k l}^{I}, B_{i j k l}^{I I}$ and $F_{i j k l}$ are given in Appendix A through Eqs. A.9 and A.10. The interfacial stress jump and average directly follow from Eq. 27. Stress concentration equations for the particular case of isotropic elasticity can be easily obtained from Appendix A or can be found in Rey and Zaoui (1980).

\subsection{Intra-crystalline slip}

Assuming $k$ is a crystallographic slip system where lattice dislocation motion may occur within both crystals I and II, then for each crystal, the plastic strain rates present in Eq. 21 yield

$$
\dot{\varepsilon}_{i j}^{p}=\sum_{k} R_{i j}^{k} \dot{\gamma}^{k}
$$

where $R_{i j}^{k}=\frac{1}{2}\left(m_{i}^{k} n_{j}^{k}+m_{j}^{k} n_{i}^{k}\right)$ is the classic Schmid (or orientation) tensor for crystallographic glide, and the summation is over $k$ (where $k=1$ to 12 for f.c.c. crystals 
like $\mathrm{Cu}$ crystals). Therefore, the dissipation density due to bulk crystallographic glide is

$$
\sigma_{i j} \dot{\varepsilon}_{i j}^{p}=\sum_{k} \tau^{k} \dot{\gamma}^{k}
$$

where $\tau^{k}=R_{i j}^{k} \sigma_{i j}$ are the resolved shear stresses, i.e. the driving forces for crystallographic slip along the active slip systems $k$.

In Eq. 21, let us now express the term $\left([\phi]-\left\langle\sigma_{i j}\right\rangle\left[\varepsilon_{i j}\right]\right) \dot{f}$ (general case) or $-\left\langle\sigma_{i j}\right\rangle\left[\varepsilon_{i j}^{p}\right] \dot{f}$ (for homogeneous elasticity). These terms characterize the intrinsic dissipation per unit volume due the shear-coupled GB migration mechanism. In the following, the link between continuum-based GB dislocation density and the coupling factor $\beta$ is detailed using the continuum dislocation density tensor introduced by Nye (1953).

\subsection{Link between GB dislocation content and coupling factor}

In the continuum dislocation theory (Nye, 1953; Kröner, 1958, 1981; Mura, 1963; Willis, 1967; Acharya, 2001), the dislocation density tensor $\alpha_{h i}$ is defined as the Curl of the incompatible elastic distortion $\beta_{j i}^{e}$ (i.e. the elastic incompatible part of the displacement gradient $u_{i, j}=\beta_{j i}=\beta_{j i}^{e}+\beta_{j i}^{p}$ in the linearized theory) as follows

$$
\alpha_{h i}=\epsilon_{h l j} \beta_{j i, l}^{e}
$$

where $\epsilon_{h l j}$ is the permutation tensor. According to the Frank-Bilby theory of surface dislocations (Frank, 1950; Bilby, 1955; Bullough and Bilby, 1956; Bullough, 1965), the plastic distortion jump (or eigendistortion) due to the GB dislocations (which is a continuum description of discrete GB defects present at the atomic scale and responsible for GB migration) can be obtained from the expression of surface dislocation densities (Bullough and Bilby, 1956; Mura, 1987) defined as follows

$$
\alpha_{h i}^{S}=\epsilon_{h l j}\left[\beta_{j i}^{e}\right] n_{l}=\epsilon_{h l j}\left(\beta_{j i}^{e I I}-\beta_{j i}^{e I}\right) n_{l}
$$

Applying the $1^{\text {st }}$ order Hadamard compatibility relation (Hadamard, 1903) at the 
discontinuity interface (i.e. $\left.\epsilon_{h l j}\left[\beta_{j i}\right] n_{l}=\epsilon_{h l j}\left(\beta_{j i}^{I I}-\beta_{j i}^{I}\right) n_{l}=0\right)$, Eq. 31 yields

$$
\alpha_{i j}^{S}=-\epsilon_{j k l}\left[\beta_{l i}^{p}\right] n_{k}
$$

Assuming $t_{l}$ a given unit vector in the boundary plane (of unit normal $n_{m}$ ) in Fig. 3 and $w_{j}$ a unit vector such as $w_{j}=\epsilon_{j m n} n_{m} t_{n}$, then the resultant Burgers vector of dislocation lines cut by $t_{l}$ is $B_{i}=\alpha_{i j}^{S} w_{j}$. Using Eq. $32, \epsilon_{j k l} \epsilon_{j m n}=\delta_{k m} \delta_{l n}-\delta_{k n} \delta_{l m}$ and $n_{n} t_{n}=0$, it comes

$$
B_{i}=-\left[\beta_{l i}^{p}\right] t_{l}
$$

The plastic distortion jump $\left[\beta_{l i}^{p}\right]$ results from plastic accommodation due the motion of gliding surface dislocation embodied by $\alpha_{i j}^{S}$. This formalism was first applied to martensitic transformations by Bullough and Bilby (1956) and later by Cahn et al. (2006b) for "shear-coupled" GB migration. If crystal II is consumed during the motion of crystal I into crystal II then crystal II will be considered as the reference lattice. This is similar to a parent phase in martensitic transformations as described in Bullough and Bilby (1956). Thus,

$$
\left[\beta_{l i}^{p}\right]=-\beta l_{i} g_{l}
$$

where $l_{i}$ and $g_{l}$ are unit vectors defined with respect to the reference crystal (see Fig. 3) so that the interface dislocations can be considered for LAGB as discrete distributions of straight dislocations parallel to a unit vector. For HAGB, the interfacial dislocations are described here as general Frank-Bilby surface dislocations (Frank, 1950; Bilby, 1955; Bullough and Bilby, 1956; Bullough, 1965). Eq. 34 characterizes a simple shear of magnitude $\beta$ defined as the "coupling factor" by Cahn and Taylor (2004) during perfect shear-coupled GB migration (i.e. without sliding). This $\beta$ factor was already identified in Subsection 2.1 without making any reference to the Frank-Bilby surface dislocation concept like in the present section.

Here, $l_{i}$ gives the direction of the Burgers vector content $B_{i}$ such that $B_{i}=B l_{i}$ 


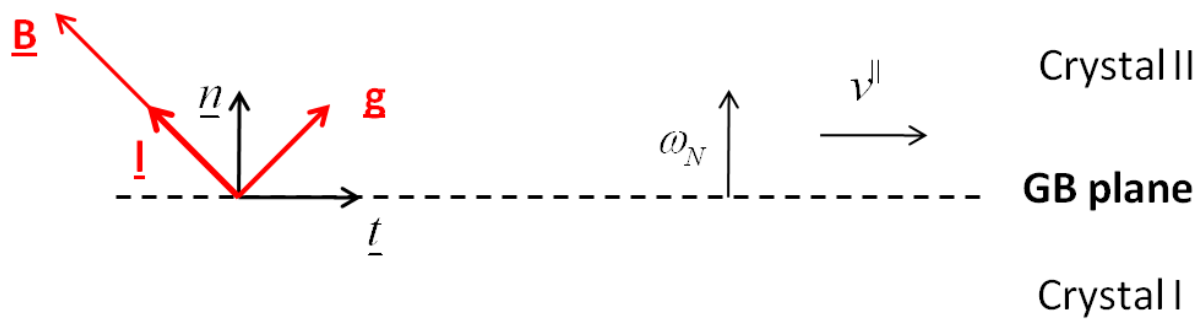

Figure 3. Definition of surface dislocation Burgers vector $B_{i}$ with respect to reference crystal II. $l_{i}$ is a unit vector in the direction of the Burgers vector, and $g_{i}$ represents the unit normal to the gliding plane of the surface dislocation.

where $B$ is its magnitude, and $g_{i}$ represents the unit normal to the gliding plane of the GB dislocations, such as Eq. 33 and Eq. 34 give

$$
B_{i}=\beta l_{i} g_{l} t_{l}=B l_{i}
$$

From the last equation, the relationship between $B$ and $\beta$ is found

$$
\beta=\frac{B}{g_{l} t_{l}}
$$

In the case of STGB with a tilt axis direction given by the unit vector $p_{j}=$ $\epsilon_{j m n} l_{m} g_{n}=\epsilon_{j m n} t_{m} n_{n}$, simple geometric considerations using Fig. 3 gives the expression of the coupling factor

$$
\beta=\frac{B}{l_{l} n_{l}}
$$

This relationship is consistent with Cahn et al. (2006a)'s work through their Eq. (21). In this section, the most important is the expression of $\left[\varepsilon_{i j}^{p}\right]$, the symmetric part of $\left[\beta_{j i}^{p}\right]$, which reads from Eq. 34

$$
\left[\varepsilon_{i j}^{p}\right]=-\widetilde{R_{i j}} \beta
$$

with

$$
\widetilde{R_{i j}}=\frac{1}{2}\left(l_{i} g_{j}+g_{i} l_{j}\right)
$$

$\widetilde{R_{i j}}$ is defined as the "orientation tensor" associated to the shear deformation (or slip) of magnitude $\beta$ coupled to GB migration. From the previous definitions of $l_{i}$ 
and $g_{j}$ in Fig. 3, $\widetilde{R_{i j}}$ and $\left[\varepsilon_{i j}^{p}\right]$ are traceless (i.e. $\widetilde{R_{k k}}=0$ ) so that the induced plastic strain due to shear-coupled GB migration is incompressible.

\subsection{Driving forces}

From Eq. 21, the total dissipation per unit volume can be rewritten in the following general form

$$
\frac{D}{V}=F_{i}\left(E_{k l}, \Sigma_{k l}, X_{j}\right) \dot{X}_{i}
$$

where $F_{i}$ are the driving forces associated to internal variables $X_{i}$. These ones depend on the overall strains $E_{i j}$ or stresses $\Sigma_{i j}$ and on the three internal variables $X_{j}$ (i.e. $\left.\varepsilon_{i j}^{p I}, \varepsilon_{i j}^{p I I}, f\right)$. In this problem, the three driving forces $F_{\varepsilon^{p I}}, F_{\varepsilon^{p I I}}, F_{f}$ associated respectively to $\varepsilon_{i j}^{p I}, \varepsilon_{i j}^{p I I}, f$ (describing three independent inelastic processes) are listed below using Eq. 21

$$
\begin{aligned}
& F_{\varepsilon^{p I}}=f \sigma_{i j}^{I} \\
& F_{\varepsilon^{p I I}}=(1-f) \sigma_{i j}^{I I} \\
& F_{f}=[\phi]-\left\langle\sigma_{i j}\right\rangle\left[\varepsilon_{i j}\right]
\end{aligned}
$$

where $\sigma_{i j}^{I}, \sigma_{i j}^{I I},\left\langle\sigma_{i j}\right\rangle,[\phi]$ and $\left[\varepsilon_{i j}\right]$ can be computed using Eq. 25 or Eq. 27. In the case of homogeneous elasticity without intra-crystalline slip, $F_{f}$ simply becomes $F_{f}=-\left\langle\sigma_{i j}\right\rangle\left[\varepsilon_{i j}^{p}\right]=\left\langle\sigma_{i j}\right\rangle \widetilde{R_{i j}} \beta=\widetilde{\tau} \beta$ following Subsection 3.3 where $\widetilde{\tau}$ is the driving resolved shear stress on the surface dislocation gliding plane associated to $\left[\varepsilon_{i j}^{p}\right], \widetilde{R_{i j}}$ is the orientation tensor previously defined in Subsection 3.3 and $\beta$ is the shear coupling factor. In the thermo-mechanics of plasticity (Rice, 1975; Maugin, 1992; Maugin and Muschik, 1994; Maugin, 2011), the critical forces (corresponding to "threshold stresses" for the previous irreversible processes) are needed to complete the theory. The considered constitutive expressions for the critical forces and the kinetics law must be chosen with respect to a positive dissipation per unit volume 
in Eq. 40.

\subsection{Critical forces for GB motion and constitutive equations}

If the critical forces for intra-crystalline slip, here denoted $F_{\varepsilon^{p I}}^{C}, F_{\varepsilon^{p I I}}^{C}$ in both crystals I and II, are higher than their respective corresponding driving forces and only the critical force for shear-coupled GB migration denoted $F_{f}^{C}$ is reached by $F_{f}$ then

$$
\begin{aligned}
& F_{\varepsilon^{p I}}<F_{\varepsilon^{p I}}^{C} \\
& F_{\varepsilon^{p I I}}<F_{\varepsilon^{p I I}}^{C} \\
& F_{f}=F_{f}^{C}
\end{aligned}
$$

In the case of homogeneous elasticity, the last equation in Eq. 42 reduces to $\widetilde{\tau}=\widetilde{\tau_{C}}$ for a given shear coupling factor $\beta$ where $\widetilde{\tau_{C}}$ is the critical shear stress resolved on the surface dislocation gliding plane. When $F_{f}$ reaches $F_{f}^{C}$ (or when $\widetilde{\tau}$ reaches $\widetilde{\tau_{C}}$ in the case of homogeneous elasticity) in Eq. 42, the GB migration flux is given by the expression of $\dot{f}$ for an infinite GB plane as follows

$$
\dot{f}=\frac{1}{V} \int_{S} \omega_{N} d S=\frac{\omega_{N} S}{V}=\frac{\omega_{N}}{L}
$$

where $L$ is the total length of the deformed bicrystal in the normal direction to the GB.

The overall Hooke's law (see Appendix A, Eq. A.6) together with Eqs. 22, 23, 43 give the following constitutive relationship between the overall stress and strain rates

$$
\dot{\Sigma}_{i j}=C_{i j k l}^{e f f}\left(\dot{E}_{k l}+\frac{\omega_{N}}{L}\left(\left[\varepsilon_{k l}\right]-S_{k l m n}^{e f f}\left[\sigma_{m n}\right]\right)\right)
$$

where $\left[\varepsilon_{i j}\right]$ and $\left[\sigma_{i j}\right]$ are respectively provided by Eq. 25 or Eq. 27 depending on homogeneous strain or stress conditions prescribed on the boundary of the bicrystal. 
Thus, for heterogeneous elastic bicrystals, Eq. 44 also writes

$$
\dot{\Sigma}_{i j}=C_{i j k l}^{e f f}\left(\dot{E}_{k l}-\dot{E}_{k l}^{p e f f}\right)
$$

where $\dot{E}_{i j}^{\text {peff }}$ is the effective (overall) plastic strain rate due to shear-coupled GB migration defined as

$$
\dot{E}_{i j}^{p e f f}=-\frac{\omega_{N}}{L}\left(\left[\varepsilon_{i j}\right]-S_{i j k l}^{e f f}\left[\sigma_{k l}\right]\right)
$$

Finally, the explicit constitutive macroscopic law is

$$
\dot{\Sigma}_{i j}=C_{i j k l}^{e f f} \dot{E}_{k l}+C_{i j k l}^{e f f}\left[\varepsilon_{k l}\right] \frac{\omega_{N}}{L}-\left[\sigma_{i j}\right] \frac{\omega_{N}}{L}
$$

If homogeneous elasticity is assumed, the last constitutive law simplifies into

$$
\dot{\Sigma}_{i j}=C_{i j k l}\left(\dot{E}_{k l}+\left[\varepsilon_{k l}^{p}\right] \frac{\omega_{N}}{L}\right)
$$

which gives, using Eq. 38 in the case where crystal II is consumed,

$$
\dot{\Sigma}_{i j}=C_{i j k l}\left(\dot{E}_{k l}-\widetilde{R_{k l}} \beta \frac{\omega_{N}}{L}\right)
$$

$\omega_{N}$ is dependent on the driving force through a mobility function which may depend on many variables such as GB misorientation, temperature, composition, etc. Following recent experimental data (Gorkaya et al., 2009), stress-driven shear-coupled GB migration exhibits a temperature dependence indicating that a thermally-activated process is at the origin of the shear-coupled GB migration. It is noteworthy that recent efforts were made to capture the kinetics law for the GB migration process at finite temperatures and strain rates by Mishin et al. (2007). Because of the limited time scale of classic MD, they used accelerated MD simulations like the parallelreplica dynamics method for a particular Cu flat $\Sigma 13(320)[001]\left(\theta=67.4^{\circ}\right)$ STGB to cover a wide GB velocity range comparable to experimental ones at a fixed temperature $(\mathrm{T}=500 \mathrm{~K})$. Through a one-dimensional analysis, they concluded that the kinetics law for stress induced-GB migration is of stick-slip nature in a large velocity range at this finite temperature. They reported the following expression for the 
tangential velocity $v^{\|}$during stick-slip dynamics

$$
v^{\|}=\frac{\tau_{c}^{0}}{\eta}\left(1-\frac{\tau_{c}}{\tau_{c}^{0}}\right)^{\frac{1}{2}} \exp \left(-\frac{\Delta G^{0}\left(1-\frac{\tau_{c}}{\tau_{c}^{0}}\right)^{\frac{3}{2}}}{k T}\right)
$$

where $\tau_{c}$ is the critical shear stress (or peak stress) at finite temperature $T$ ( $\tau_{c}^{0}$ is the critical shear stress at $0 \mathrm{~K}), \eta$ is an effective friction coefficient, $\Delta G^{0}$ is the stress-free activation energy barrier and $k$ is the Boltzmann constant. Eq. 50 was found to work in a large range of velocities $v^{\|}$from $5 \times 10^{-4}$ to $5 \mathrm{~m} / \mathrm{s}$ (Mishin et al., 2007). Thus, the GB migration flux at finite temperature in the model could be determined from Eq. 50. However, the accurate determination of the kinetics parameters for the three investigated $\mathrm{Cu}$ STGB will need specific simulation methods (parallel-replica dynamics, nudged elastic band methods) which are out of the scope of the present study.

Eq. 47 shows that once GB migration is active for a given normal velocity $\omega_{N}$ (which also depends on the applied velocity to the bicrystal), the instantaneous stress decrease due to induced plastic strain is dependent on the effective elastic moduli $C_{i j k l}^{e f f}$ (or $C_{i j k l}$ in the case of homogeneous elasticity), the GB character (through $\widetilde{R_{i j}}$ and $\beta$ ) and the bicrystal finite size $L$. The calculation of $\dot{f}$ (or equivalently the volume fraction increment dictated by the stepwise normal GB motion at each GB migration event) will be specified and discussed in Section 4 (for 0K and 500K temperatures) with application to particular [001] Cu STGB. For the latter, the atomistic mechanisms and collective steps when GB migrates are known following appropriate vectors of the DSC lattice (Rae and Smith, 1980; Cahn et al., 2006a). 


\section{Application to $\mathrm{Cu}$ [001] STGB}

\subsection{Shear coupling modes}

Following Cahn et al. (2006a,b); Mishin et al. (2007); Zhang et al. (2008), we considered [001] STGB in cubic metals like Cu (f.c.c. metal). GB are generally characterized by five angles. Four angles are set up by choosing the tilt axis and the GB plane is a particular mirror plane of the bicrystal containing the tilt axis. The misorientation angle $\theta$ is defined as the tilt angle between the [100] directions of both crystals in the counterclockwise direction, with $0<\theta<\pi / 2$ due to the four-fold symmetry around the tilt axis. Hence, the GB plane lies along the bisector between the [100] directions like in Fig. 4.

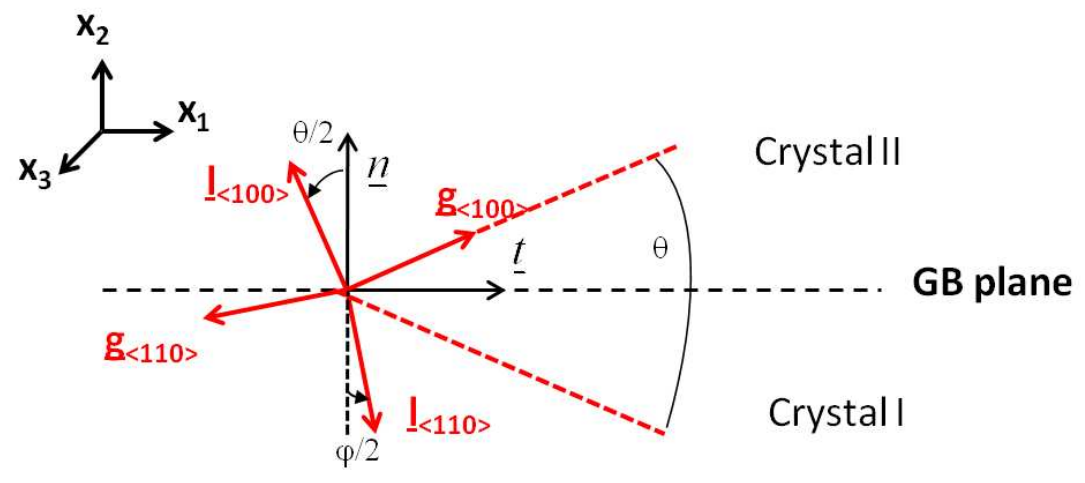

Figure 4. Definition of the misorientation angle $\theta$ for [001]-type tilt boundaries. Two Burgers vectors $B_{i}$ with directions given by unit vectors $l_{i}$ are possible which correspond to two different mappings for Burgers circuit. The normal directions to the slip planes are given by unit vectors $g_{i}$. Angles $\theta$ and $\varphi$ are linked each other by $\varphi=\pi / 2-\theta$.

Following Cahn et al. (2006b), two mappings for the Burgers circuit allows two possible $B_{i}$ and two associated "coupling modes" to be defined. The first "coupling mode" called the $<100>$ mode (or "mode I") is such that $l_{i}$ is parallel to the cube direction [010] of the reference lattice (crystal II) and the Frank-Bilby dislocation 
slip planes are (100) (Cahn et al., 2006b). The slip plane is represented to the left of the GB normal in Fig. 4. For small $\theta$ (LAGB), the expressions for $B_{i}$ and the associated dislocation density can be resolved by a discrete distribution of single lattice dislocations of Burgers vectors $b_{i}=a_{L}[010]$ where $a_{L}$ is the lattice parameter (Bullough, 1965). For LAGB, the critical stress is proportional to the glide component of the Peach-Koehler force required to initiate the collective glide of the arrays of GB dislocations (Molodov et al., 2007). There are two kinds of LAGB: either for small $\theta$ or for $\theta$ close to $\pi / 2$ (i.e. $\varphi=\pi / 2$ - $\theta$ near 0$)$. The latter corresponds to the GB "mode II" migration ( $<110>$ mode) where $l_{i}$ is parallel to the direction $[1 \overline{1} 0]$ of the reference lattice (crystal I) and the associated resolved single dislocations are of type $b_{i}=a_{L} / 2[1 \overline{1} 0]$. In this case, the Frank-Bilby surface dislocation glides along (110) planes, which is represented to the right of the GB normal in Fig. 4.

For HAGB, the discrete distributions of single dislocations can not be resolved anymore (Li, 1961) and the Frank-Bilby equation (Eq. 32) is here used for these STGB. Disclination models would be possible to describe HAGB (Li, 1972; Clayton et al., 2006; Upadhyay et al., 2011). These approaches are based on different kinematics and couple-stress fields which are not discussed in the present paper. As established by Cahn et al. (2006b), the Frank-Bilby equation (FBE) introduced in Subsection 3.3 provides two feasible solutions for [001] STGB and their continuous GB dislocation density. These two solutions (as functions of $\theta$ ) correspond to Burgers vectors either parallel to [010] (denoted as $B_{i}<100>$ for "mode I") or parallel to [11̄0] (denoted as as $B_{i}<110>$ for "mode II"). Here, [010] and [11̄0] are crystallographic directions respectively defined in the crystal II and crystal I. For GB "mode I" migration, the consumed grain is crystal II (reference lattice) such as $\dot{f} \geq 0$, whereas for "mode II" migration, crystal I (reference lattice) is consumed with $\dot{f} \leq 0$. Under simple shear loading, these two coupling modes compete with each other, and the transition from one mode to the other occurs at a critical misorientation angle $\theta$ which depends on 
temperature (see Fig 8 in Cahn et al. (2006a)). Note that as the temperature drops, the $\theta$ range of "mode II" expands and it may be the only active coupling mode for all values of $\theta$ at $\mathrm{T}=0 \mathrm{~K}$. The invoked reason is that the activation of "mode I" requires the breaking of the mirror symmetry due to equivalent row translations by lattice vectors $1 / 2[001]$ and $1 / 2[00 \overline{1}]$. This symmetry can only be broken at finite temperatures where ledges and other defects may form easily. This issue was also checked using the gamma-surfaces associated to both modes at 0K by Cahn et al. (2006a)

Thus, due to the complexity of atomistic mechanisms in the case of general GB, we here limit atomistic investigations to study the constitutive behaviour associated with the STGB shear-coupled migration. For this case, MD simulations are conducted for three $\mathrm{Cu}$ [001] STGB with misorientation angles $\theta=77.32^{\circ}, 53.13^{\circ}$, $28.07^{\circ}$ which show the well-identified temperature dependent shear-coupling $<100>$ and $<110>$ modes linked to two characteristic coupling factors $\beta$ (resp. positive and negative). According to Cahn et al. (2006a), the coupling factor $\beta$ depends on $\theta$ and on the "coupling modes" as follows

$$
\begin{aligned}
& \beta_{<100>}=2 \tan \left(\frac{\theta}{2}\right) \\
& \beta_{<110>}=-2 \tan \left(\frac{\varphi}{2}\right)
\end{aligned}
$$

where $\varphi=\frac{\pi}{2}-\theta$. MD simulations shown in Subection 4.2 will first provide the shear stress response, the temperature dependent shear coupling mode as a function of the GB character, the critical shear stresses (or "peak stresses"), the stress accommodation due to shear (shear stress drop during GB migration) and the saw-tooth behaviour (stick slip character). Second, the results of the developed micromechanicsbased model regarding effective elastic moduli, shear stress drops and dissipated energy will be discussed in Subection 4.3. The roles of GB character, bicrystal size and anisotropic elasticity will be studied in Subection 4.3. 


\subsection{Molecular dynamics simulations}

\subsubsection{Computational methodology}

In this section, a few "flat" $\mathrm{Cu}$ [001] STGB, namely $\Sigma 41(540)\left(\theta=77.32^{\circ}\right), \Sigma 5(210)$ $\left(\theta=53.13^{\circ}\right), \Sigma 17(410)\left(\theta=28.07^{\circ}\right)$, were studied at $0 \mathrm{~K}$ and at $500 \mathrm{~K}$ temperatures using the EAM interatomic potential provided by Mishin et al. (2001) for Cu. Note that in the work of Cahn et al. (2006a) where MD simulations were performed under simple shear loading at 800K, the first two STGB display "mode II" migration, and the last one displays the "mode I" (see table 1 in Cahn et al. (2006a)). However, all of them display the "mode II" ( $<110>$ mode) at 0K as can be inferred from the plots in their Fig 8. Here, each bicrystal with [001] STGB is created using the coincident site lattice (CSL) model by placing the first crystal on the top of the other using the following procedure (Fig. 5).

The tilt axis (x3-axis) is along [001] direction. The horizontal plane (x1,x3) corresponds to the GB plane, and [100] directions for crystals I and II makes an angle $\theta$. Several initial configurations are also tested by shifting the upper grain with respect to the lower along the (x1) direction so as to obtain the lowest energy state of a GB configuration after atomic relaxation. The energy minimization is performed with the LAMMPS simulator ${ }^{2}$ using a conjugate gradient method. The methodology used to construct the bicrystal in a relaxed configuration (Fig. 6) is similar to what has been done before by several authors (Cahn et al., 2006a; Spearot et al., 2008). The methodology adequately accounts for in-plane shifts and result in a relaxed configuration with GB structural units same as those obtained by previous authors. Similarly, interfacial energy values are also in excellent agreement with previously published results given in Spearot et al. (2008). Each Cu crystal for sim-

$\overline{2}$ LAMMPS Molecular Dynamics Simulator; http///lammps.sandia.gov/. 


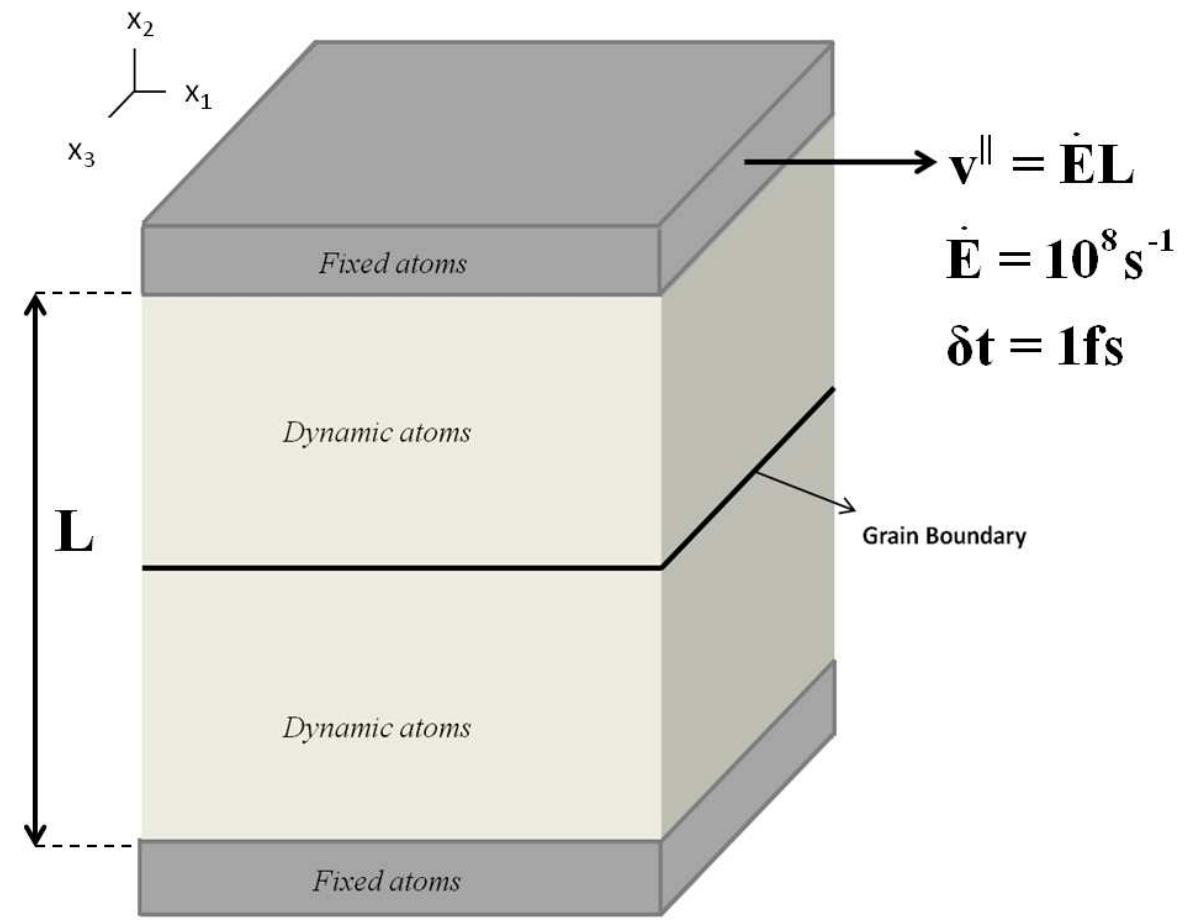

Figure 5. Schematic figure of the computational atomistic unit cell box with coordinate axes and periodic boundary conditions. $L$ is the total length in the normal direction (as defined in the text) to the GB plane for dynamic atoms subjected to shear.

ulations is approximately cubic shaped, and the simulation block for the bicrystal contains between 30000 - 45000 atoms with periodicity in the (x1) and the (x3) directions. System dimensions chosen were adequate for the problem considered and with number of atoms very similar to those reported by Cahn et al. (2006a). Periodic dimensions in the $(\mathrm{x} 1)$ and the $(\mathrm{x} 3)$ directions are listed as follows: $4.7 \times$ $4.7 \mathrm{~nm}$ for $\Sigma 41(540)\left(\theta=77.32^{\circ}\right), 3.3 \times 3.3 \mathrm{~nm}$ for $\Sigma 5(210)\left(\theta=53.13^{\circ}\right)$, and, $6 \times$ $5.8 \mathrm{~nm}$ for $\Sigma 17(410)\left(\theta=28.07^{\circ}\right)$. After molecular static simulation to obtain optimized structure, the system was further equilibrated using Molecular Dynamics (MD) simulations for 40 ps at 0.001 ps under isothermal-isobaric NPT ensemble. Temperature was increased in increments and for every $100 \mathrm{~K}$ temperature increase (i.e. $100 \mathrm{~K}, 200 \mathrm{~K}, 300 \mathrm{~K}, 400 \mathrm{~K}$ and $500 \mathrm{~K}$ ), the system was further equilibrated for 40ps each with 1 fs time step. MD simulations for shear loading were performed in 
the canonical NVT ensemble with Nose-Hoover thermostat under a fixed volume.

The structure is subjected to constant shear strain rate loading as follows.

$\Sigma 5(210)$

$\Sigma 17\left(\begin{array}{lll}4 & 1 & 0\end{array}\right)$

$\Sigma 41(540)$

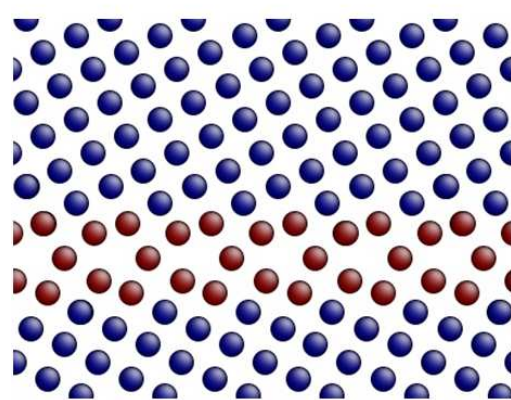

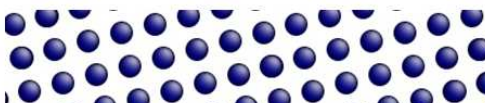
1000000000001 000.0000000 .000 0.000 .000 .000$. 000000000000

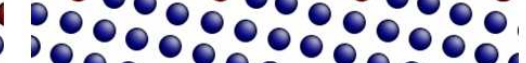
10000000000 100000000000

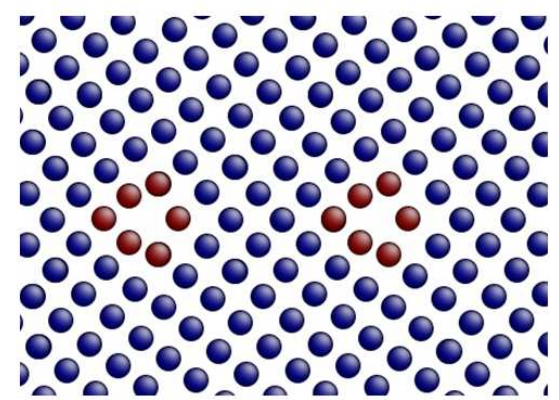

[210]

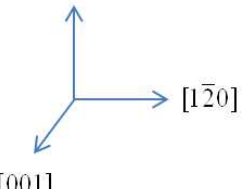

[001]
[410]

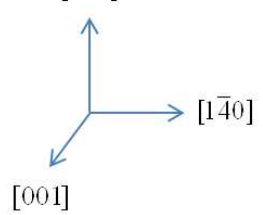

[540]

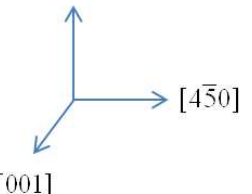

[001]

Figure 6. Structure of the Cu bicrystal showing the STGB Structural units from left to right: $\Sigma 5(210)\left(\theta=53.13^{\circ}\right), \Sigma 17(410)\left(\theta=28.07^{\circ}\right), \Sigma 41(540)\left(\theta=77.32^{\circ}\right)$. Crystallographic orientation of the lower crystal are shown at the bottom.

The simulation block (Fig. 5) is sandwiched between the top and the bottom layer (along the $\left(\mathrm{x}_{2}\right)$ axis) of thickness about 2 times the potential cut-off distance. These two layers do not participate in computing data from the simulations and serve only to impose simple shear loading. The bottom block is held fixed and the constant shear velocity $v_{0}=v^{\|}=\dot{E} L$ is applied to the top part of the block in the $\left(\mathrm{x}_{1}\right)$ direction, where $\dot{E}$ is the constant shear strain rate $\left(\dot{E}=10^{8} s^{-1}\right)$ and $L$ is the simulation block length containing unconstrained atoms. Here, two different values were chosen to keep initial crystal characteristic sizes lower or equal to $10 \mathrm{~nm}$ to mimic nanocrystals, namely $L=12.2 \mathrm{~nm}$ and $L=20 \mathrm{~nm}$. The time step is $1 f_{s}$. These simulations are conducted at $0.001 \mathrm{~K}$ and at 500K, and the overall stress tensor was computed using the standard virial expression averaged over all dynamic atoms. The GB position was also tracked from the common neighbour analysis (CNA) computation. The CNA value for atoms in f.c.c. lattice is 1 and for atoms forming 
GB structural units it is 5 (Faken and Jonsson, 1994; Tsuzuki et al., 2007). For higher temperature $(500 \mathrm{~K})$ CNA values averaged over 100 time steps (and centered around the time step they were averaged) were computed due to thermal fluctuation. Therefore, a threshold value of 4.9 was used to locate the position of the grain boundary at $500 \mathrm{~K}$. For $0.001 \mathrm{~K}$, threshold value of 5 was used to locate the GB position.

\subsubsection{Stick-slip behaviours at $0 K$ and $500 K$}

As shown by the MD simulation results (Fig. 7) performed at $0.001 \mathrm{~K}(\sim 0 \mathrm{~K}$ i.e. very close to the static case), the stress induced shear-coupled GB migration for these Cu STGB is of stick-slip nature. The saw-tooth shape of the shear stress responses reported on Fig. 7 characterizes this stick-slip behaviour. This behaviour is similar to serrated plastification due to collective motion of bulk dislocations in different alloys which is characterized by intermittent bursts (Chihab et al., 1987; Kubin et al., 2002). Here, the stick slip behaviour due to GB migration is due to intermittent collective motion of GB dislocations as described in Subsection 4.1. These shear stress - shear strain curves represent the typical behaviour at $\mathrm{T}=0 \mathrm{~K}$. The most important features are the GB character-dependent effective elastic shear moduli for bicrystals, the critical shear stresses for GB migration identified as the peak shear stresses in Fig. 7 and the shear stress drops (or the lower stresses after peak stresses). All these parameters are (quasi)-identically repeated during shear deformation. In this paper, these features will be analyzed in Subsection 4.3 in the light of the developed micromechanics-based approach. 


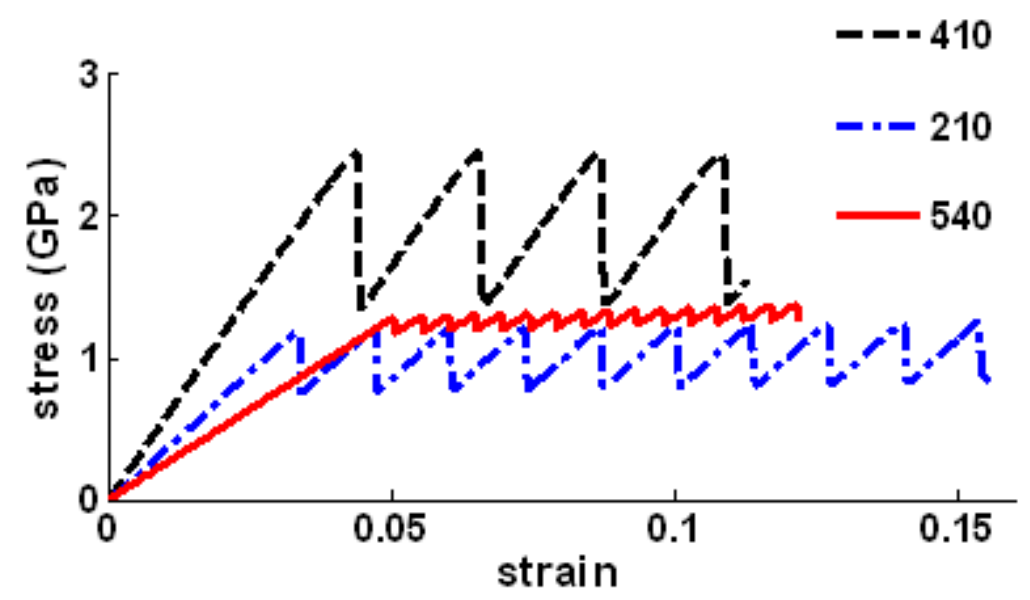

Figure 7. Shear stress vs. strain curves at 0K for $\mathrm{Cu} \Sigma 17(410), \Sigma 5(210), \Sigma 41(540)$ [001] STGB.

Fig. 8a shows the shear stress vs. strain curves for $\mathrm{Cu} \Sigma 5(210)$ at both $0 \mathrm{~K}$ and $500 \mathrm{~K}$ temperatures. The critical shear stress is lowered when the temperature increases and important stress fluctuations at $500 \mathrm{~K}$ are due to atomic thermal vibrations. However, the bicrystal responses in terms of elastic shear moduli and shear stress drop amplitude are not really sensitive to temperature change. It is noteworthy that the obtained shear stress drop is consistent with Cahn et al. (2006a)'s work (see their Fig 9) even though the simulation bicrystal size $L$ may be different. Fig. 8 b reports the evolution of GB position during the mechanical test as a function of applied shear strain. At each discrete migration event, a jump of GB position is clearly seen at both temperatures. Furthermore, the negative motion direction with respect to the $\left(\mathrm{x}_{2}\right)$ axis means that for this STGB, the shear-coupled GB motion always follows the same "mode II" $(<110>$ mode $)$.

Fig. 9a shows the shear stress vs strain curves for $\mathrm{Cu} \Sigma 41(540)$ at both $0 \mathrm{~K}$ and 500K temperatures. Fig. 9b reports the evolution of GB position as a function of applied shear strain. GB position jumps along the negative $\left(\mathrm{x}_{2}\right)$ direction are clearly seen at both temperatures. Again the shear stress drop magnitude at each GB mi- 

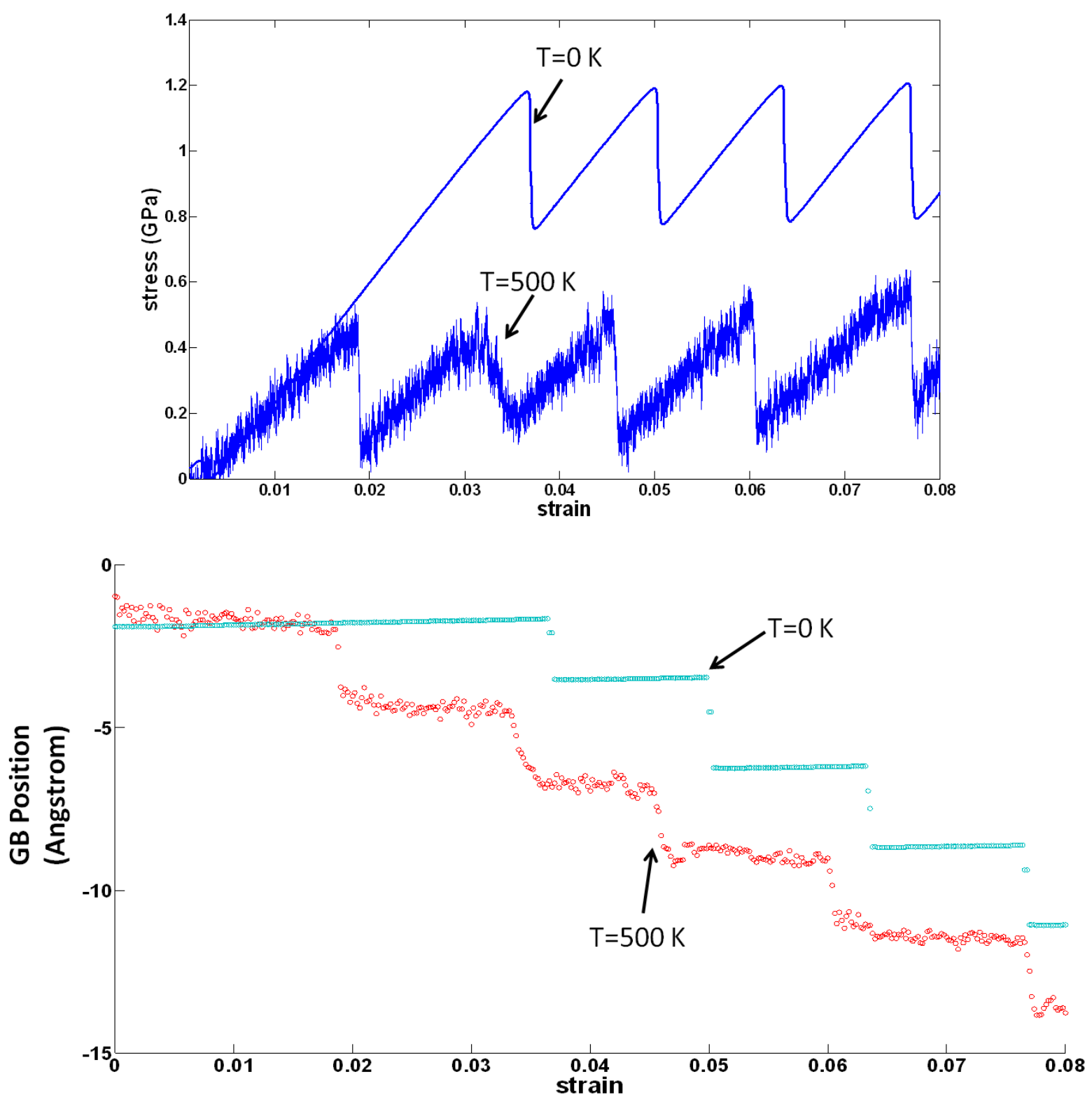

Figure 8. Shear stress vs. shear strain curves for $\mathrm{Cu} \Sigma 5$ (210) [001] STGB at 0K and 500K temperatures (a), Simultaneous record of GB position (in Angström) as a function of shear strain at $0 \mathrm{~K}$ and $500 \mathrm{~K}(\mathrm{~b})$.

gration event does not seem to be affected by finite temperature in strong contrast with the peak stress at migration activation. Note that for both temperatures, only the $<110>$ mode is present at both temperatures. These results are consistent with Cahn et al. (2006a)'s work. 

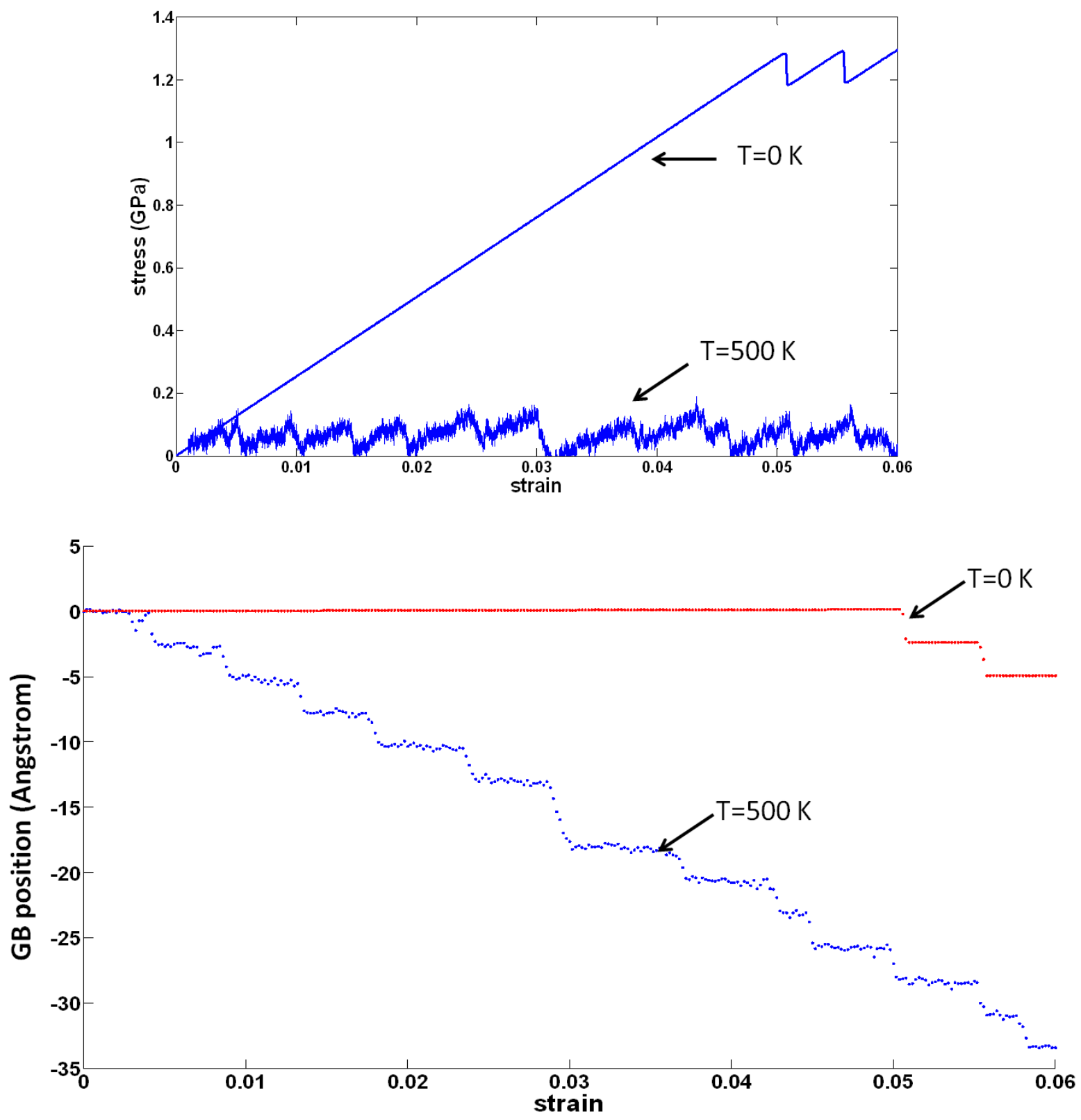

Figure 9. Shear stress vs. shear strain curves for $\mathrm{Cu} \Sigma 41(540)$ [001] STGB at 0K and 500K temperatures (a), simultaneous record of GB position (in Angström) as a function of shear strain at $0 \mathrm{~K}$ and $500 \mathrm{~K}(\mathrm{~b})$. 


\subsubsection{Bicrystal size effect on stress drop}

We considered $\mathrm{Cu}[001] \Sigma 5(210), \Sigma 17(410)$ and $\Sigma 41(540)$ STGB at $0 \mathrm{~K}$ to study the effect of the size of bicrystal on their shear stress response. It is first observed that the peak stress and the elastic shear moduli are not really sensitive to the size $L$ of the deformed bicrystal in the direction normal to the GB plane (Fig. 10). In contrast, the shear stress drop is very sensitive to $L$. This trend was also reported by Ivanov and Mishin (2008) for $\mathrm{Al}[112] \Sigma 21(\overline{2} 4 \overline{1})$ STGB at finite temperature (T=300K). To

our knowledge, we did not find anymore STGB examples in the literature treating these size effect on the shear stress drop. More specifically, it is seen that the shear stress drop magnitude scales approximately with the inverse of $L$. This trend will be further analyzed in Subsection 4.3 as an application of the micromechanics-based model. Lastly, it is observed that the shear coupling "mode II" migration is always active at $0 \mathrm{~K}$ and does not depend on $L$.

\subsubsection{Dual temperature dependent behaviour}

Fig. 11 shows the plots of GB position vs. shear strain for $\mathrm{Cu} \Sigma 17(410)$ at $0 \mathrm{~K}$ and at $500 \mathrm{~K}$ temperatures. Note that at $0 \mathrm{~K}$ temperature, shear-coupled GB migration exhibits the $<110>$ mode (with negative $\beta$ coupling factor) whereas at $500 \mathrm{~K}$, it now exhibits the $<100>$ mode (with positive $\beta$ coupling factor). This result is seen at $0 \mathrm{~K}$ in Fig. 11a by a negative GB motion along $\left(\mathrm{x}_{2}\right)$ with respect to a positive applied shear at the top of the bicrystal along $\left(\mathrm{x}_{1}\right)$. At 500K, Fig. 11b reports a positive GB motion along $\left(\mathrm{x}_{2}\right)$ for same positive shear along $\left(\mathrm{x}_{1}\right)$. In Fig. 11c, it is first observed at $500 \mathrm{~K}$ that GB migration occurs earlier compared to 0K. Hence, the same trend as the two other STGB is observed (see Figs. 8b and 9b). In addition, 


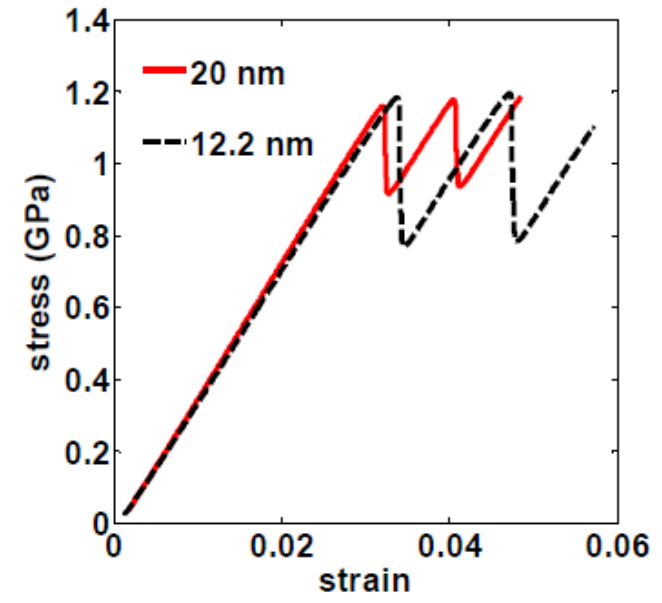

(a)

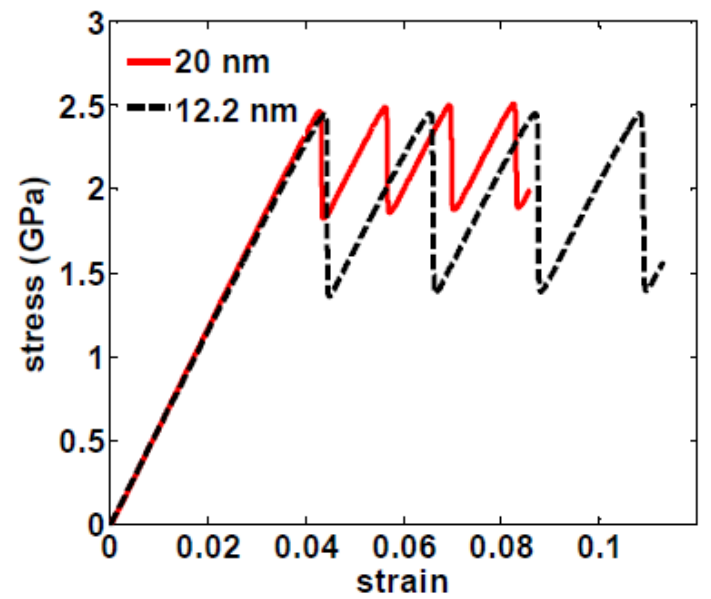

(b)

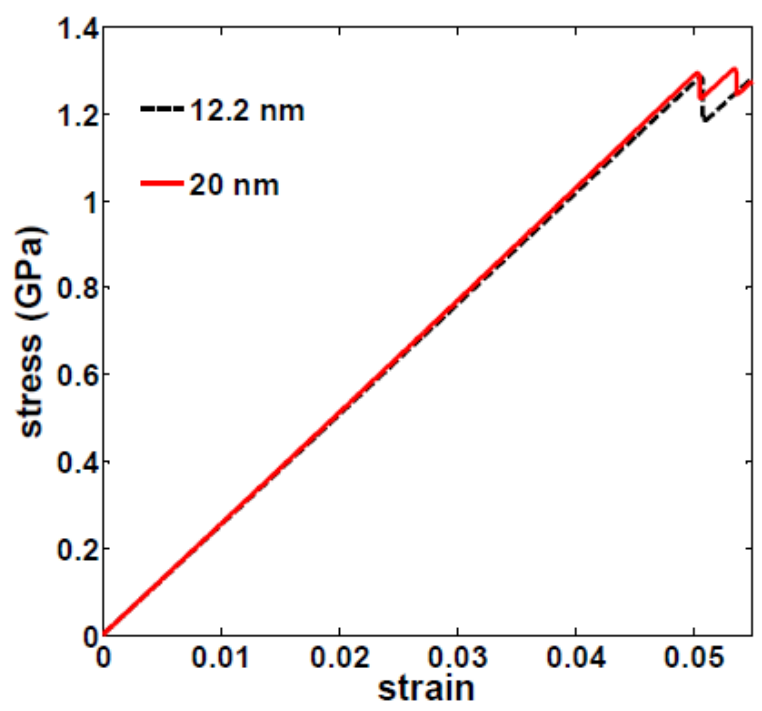

(c)

Figure 10. Shear stress vs. shear strain curves at $0 \mathrm{~K}$ with two bicrystal size $L=12.2 \mathrm{~nm}$ and $20 \mathrm{~nm}$ for $\mathrm{Cu} \Sigma 5(210)$ (a), $\Sigma 17(410)$ (b) and $\Sigma 41(540)$ (c).

the GB steps follow opposite directions and different magnitudes between two subsequent GB migration events which reveal the presence of different GB slip modes. 

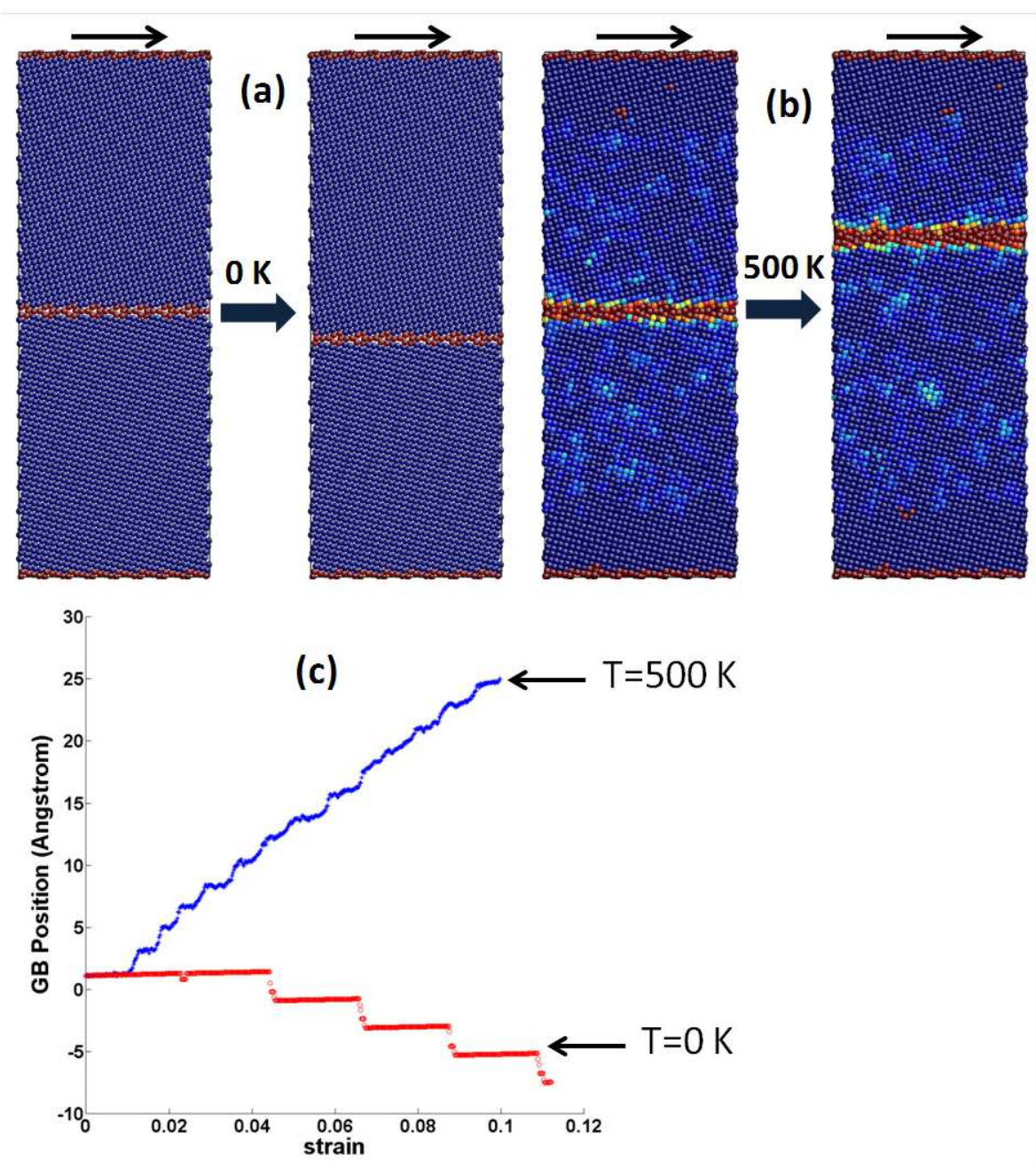

Figure 11. Snapshots of shear-coupled GB migration for Cu $\Sigma 17(410)$ STGB: at 0K from 0 to 0.11 shear strain (a), at $500 \mathrm{~K}$ from 0 to 0.099 shear strain (b), STGB position (in Angström) vs. shear strain at $0 \mathrm{~K}$ and $500 \mathrm{~K}$ (c).

\subsection{Model results and comparisons with atomistic simulations}

The micromechanics-based approach is applied to the three previously investigated Cu bi-crystals with [001] STGB. The motivation to study $\mathrm{Cu}$ bicrystals lies in the fact that they exhibit a strongly anisotropic elastic behaviour characterized by the following anisotropic coefficient (Hirth and Lothe, 1982): $a=\frac{2 C_{44}}{C_{11}-C_{12}}=3.26$. The cubic elastic moduli for $\mathrm{Cu}$ are taken as $C_{11}=170 \mathrm{GPa}, C_{12}=122.5 \mathrm{GPa}$ and $C_{44}=76$ GPa. These elastic constants are given by Mishin et al. (2001) and were used by these 
authors to validate the EAM potential for $\mathrm{Cu}$. In this paper, the application of the micromechanics-based theory is mainly focused on the effect of elastic anisotropy on the shear stress-strain curves before and at the first shear-coupled GB migration event (Fig. 12).

In the recent contribution of Luque et al. (2010), these authors did not consider the effective elastic properties of the bicrystal in the computation of the macroscopic plastic strain induced by shear-coupling GB migration (see their Eq. (2)). Furthermore, the so called "orientation factor" in the same equation is not defined as it is here through Eq. 39. In the following, we show that this strong elastic anisotropy results in elastic shear moduli (before migration events), shear stress drops and dissipated energy that are very sensitive to the GB character. The results obtained by atomistic simulations performed at $0 \mathrm{~K}$ obtained in subsection 4.2 serve as benchmarks for the predictions of the micromechanical formulation.

The explicit formulas obtained by the micromechanical scheme are developed for general heterogeneous elasticity (see Appendix A) and can be applied to isotropic elasticity and to anisotropic elasticity for [001] STGB (see Appendix B). The discrepancies between both elastically isotropic and heterogeneous formulations on the effective elastic moduli, the shear stress drops and the dissipated energy when shearcoupled migration occurs are highlighted in comparison with the MD results. The values of shear stress drop magnitudes for different bicrystal sizes are also reported and discussed.

\subsubsection{On effective elastic shear moduli}

For homogeneous isotropic elastic properties, the isotropic elastic coefficients for $\mathrm{Cu}$ 


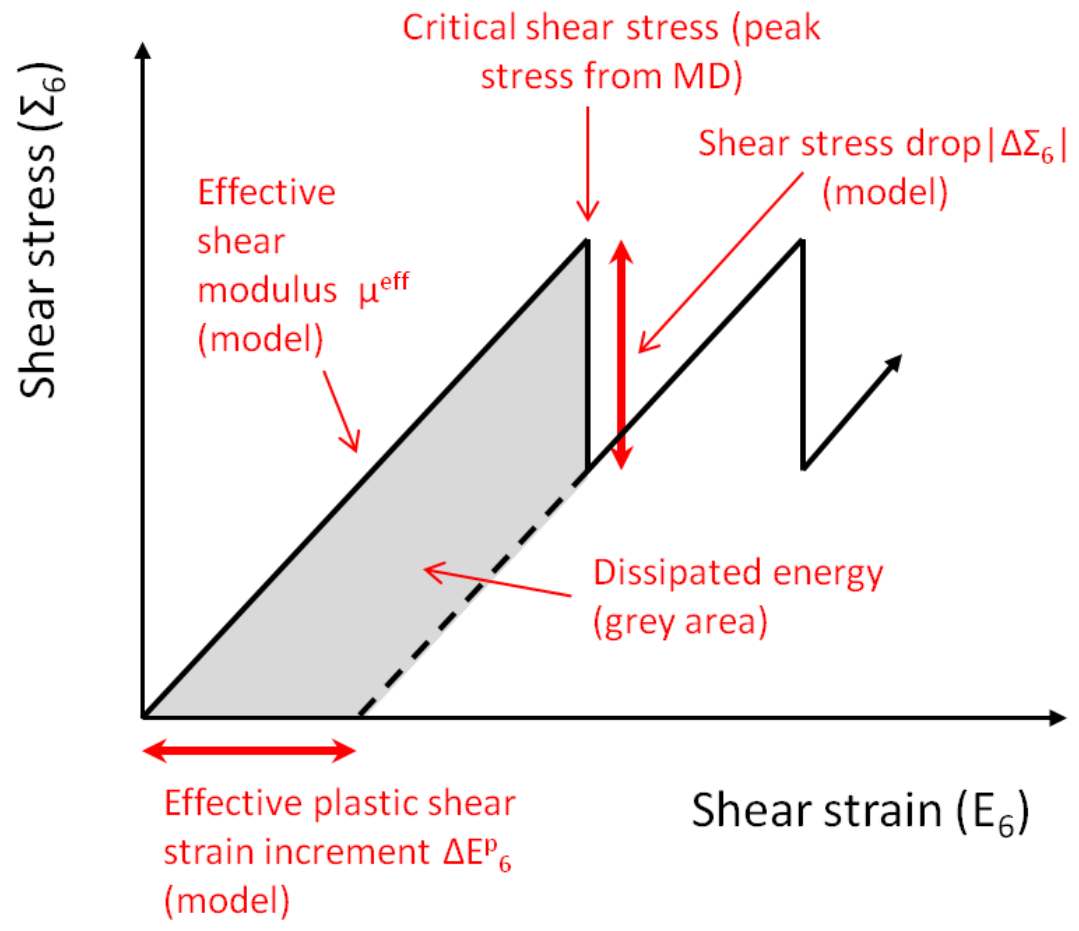

Figure 12. Schematic representation of the shear stress vs. shear strain curve and the quantitative values predicted by the micromechanical model: effective elastic shear moduli, shear stress drop, dissipated energy. The critical shear stress (also called "peak stress") is obtained by MD simulations.

are obtained using the classic Voigt-Reuss-Hill average model (Voigt, 1928; Reuss, 1929; Hill, 1952). This model gives $\mu=47.8 \mathrm{GPa}$ (isotropic elastic shear modulus) and $\nu=0.345$ (Poisson ratio). The isotropic shear modulus is reported on Table 1 for comparisons with the effective elastic shear moduli derived for general elastic anisotropy. The application of Appendix B gives the numerical values for elastic moduli in both crystals using the cubic symmetry for $\Sigma 41(540)\left(\theta=77.32^{\circ}\right), \Sigma 5(210)$ $\left(\theta=53.13^{\circ}\right), \Sigma 17(410)\left(\theta=28.07^{\circ}\right)$ that can be used to derive the effective elastic moduli (Eq. A.7 in Appendix A) for the three investigated bicrystals. We find for $f=1-f$ (initial effective elastic shear moduli) that the effective elastic shear moduli (Table 1) are in good agreement with the atomistic simulations at $0 \mathrm{~K}$ and at $500 \mathrm{~K}$. In contrast, we remark that the classic isotropic assumption obtained from VoigtReuss-Hill average is sometimes far from the atomistic results for the studied $\mathrm{Cu}$ 
[001] STGB, which means that the simple elastic isotropic assumption is not realistic for $\mathrm{Cu}$ bicrystals. It is noteworthy that after a few GB migration events (e.g. four events like the ones present in Fig. 7), the effective elastic shear moduli repeat quasi-identically. This is due to the fact that the total GB displacement after four events is very small compared to $L$. This trend can be explained by the model since $f$ still remains close to $1-f$ in Eq. A.7. Conversely, when one of both crystals is totally consumed by the other (the study was not investigated due to large CPU times for atomistic simulations), the micromechanical model is able to predict a change in the effective elastic moduli when $f$ or $1-f$ tends to zero in Eq. A.7, depending on the shear coupling mode.

\begin{tabular}{cccc}
\hline Effective elastic shear moduli (GPa) & $\Sigma 41(540)$ & $\Sigma 5(210)$ & $\Sigma 17(410)$ \\
\hline Atomistic simulations & 25.4 & 35.6 & 57.4 \\
\hline Present model (anisotropic) & 25.7 & 39.5 & 61.8 \\
\hline Table 1 & 47.8 & 47.8 & 47.8 \\
\cline { 2 - 4 }
\end{tabular}

Elastic shear moduli obtained by the MD results and by the present micromechanical model accounting for anisotropic elasticity for $\Sigma 41(540), \Sigma 5(210), \Sigma 17(410)$ [001] STGB. The isotropic Voigt-Reuss-Hill average is also given for comparisons.

\subsubsection{On overall shear stress drops and dissipated energy due to shear-coupled GB migration}

The mechanical responses given by the micromechanical model can be enriched by the critical forces $F_{f}^{C}$ or the critical resolved shear stresses on the GB slip plane $\widetilde{\tau_{C}}$ 
in Eq. 42 directly obtained from the peak stresses (or critical shear stresses $\tau_{C}$ ) of the atomistic results (see Subsection 4.2). These peak stresses resulted from simple shear performed at an applied material velocity $v^{\|}$parallel to the GB plane (see Figs. 5 and 7$)$.

In this part, we focus on the analysis of shear stress drops and dissipated energy in the light of the micromechanical approach when the first GB migration event occurs. Hence, we set $f=1-f$ in the constitutive model assuming either isotropic elasticity or fully anisotropic elasticity in both crystals. We start by the analysis of first shear stress drops when the "mode II" ( $<110>$ mode) is activated at $0 \mathrm{~K}$ for the three GBs and at $500 \mathrm{~K}$ for $\Sigma 41(540)$ and $\Sigma 5(210)$. Then, the model is applied to the case when the "mode I" (<100> mode) is activated at $500 \mathrm{~K}$ for $\Sigma 17(410)$. Lastly, the model is applied to understand the origin of bicrystal size effects on stress drops at $0 \mathrm{~K}$ for the three STGBs.

As seen from atomistic simulations, "mode II" is a dominating shear mode especially at $0 K$ for the three GBs. According to Fig. $4, l_{i}$ and $g_{i}$ are defined so that the $<110>$ mode ("mode II") is characterized by $l_{i} n_{i}=g_{i} t_{i}=-\cos \left(\frac{\varphi}{2}\right)=-\cos \left(\frac{\pi}{4}-\frac{\theta}{2}\right)$ which gives $l_{i}=\left(\sin \left(\frac{\pi}{4}-\frac{\theta}{2}\right),-\cos \left(\frac{\pi}{4}-\frac{\theta}{2}\right), 0\right), g_{i}=\left(-\cos \left(\frac{\pi}{4}-\frac{\theta}{2}\right),-\sin \left(\frac{\pi}{4}-\frac{\theta}{2}\right), 0\right)$ and $\widetilde{R_{i j}}$ from Eq. 39. Since crystal I is the parent grain, only crystal II undergoes plastic deformation, thus $\left[\varepsilon_{i j}^{p}\right]=\varepsilon_{i j}^{p I I}=-\widetilde{R_{i j}} \beta$. When the critical shear stress is reached, shear-coupled GB migration is active. Then, time integration of Eq. 47 during the first shear-coupled GB migration event yields

$$
\dot{\Sigma}_{i j} \delta t=C_{i j k l}^{e f f} \dot{E}_{k l} \delta t+\frac{h_{N}}{L}\left(C_{i j k l}^{e f f}\left[\varepsilon_{k l}\right]-\left[\sigma_{i j}\right]\right)
$$

From atomistic results, the time step $\delta t$ for each single shear-coupled GB migration event is a few ps. This time scale is out of the scope of continuum mechanics for which GB migration is seen as instantaneous. In Eq. 52, the strain and stress jumps 
(resp. $\left[\varepsilon_{i j}\right]$ and $\left[\sigma_{i j}\right]$ ) can be computed using the expressions for strains and stresses given in Appendix A for homogeneous stress or strain boundary conditions. These jumps depend on the elastic properties of both crystals as given in Appendix B and depend on $\widetilde{R_{i j}}$ and $\beta$. In Eq. 52, $h_{N}$ denotes the normal step height during stepwise GB motion (at the first GB migration event) which corresponds to the characteristic step height due to GB disconnection loop nucleation (Hirth and Pond, 1996; Hirth et al., 2007; Pond et al., 2008; Wang et al., 2011). This mechanism is supposed to be present for the investigated STGB which are all considered as HAGB (since their misorientation is higher than $10^{\circ}$ ) (Cahn et al., 2006a). In the case of "mode II", the GB plane moves down (negative motion with respect to the $\left(\mathrm{x}_{2}\right)$ axis in Fig. A.1) to a new position when $\tau_{C}$ is reached for which the activation energy for GB migration is overcome. The stored energy released at each increment of the GB motion is immediately dissipated and the temperature returns to initial temperature so that an isotherm assumption can be justified (this would not be the case for materials problem with slow energy dissipation). In the present formulation, the second term in Eq. 52, that contains the strain and stress jumps, $h_{N}$ and the effective elastic moduli, is responsible for shear stress drop when migration is active. Without trying to determine the complete activation energy profile for the three studied STGB (like Eq. 50), the transition from an unstable state to a metastable state associated with the dissipative GB migration event is then described by a normal step height $h_{N}$. This characteristic distance $h_{N}$ is also linked to the DSC lattice vector for coincident (CSL) GB. The DSC lattice is the largest lattice including all the sites of the lattices of both crystals. According to Rae and Smith (1980), King and Smith (1980), Cahn et al. (2006a), the disconnection step height $h_{N}$ is linked to the DSC lattice spacing. Thus, the expression of the disconnection step height $h_{N}$ depends on the GB character (through $\theta$ ) and on the lattice parameter $a_{L}$. For the $<110>$ 
mode, $h_{N}$ is negative and is given by (Cahn et al., 2006a)

$$
h_{N}^{<110>}=-\frac{a_{L}}{\sqrt{2}} \cos \left(\frac{\varphi}{2}\right)
$$

where $a_{L}=0.3615 \mathrm{~nm}$ for $\mathrm{Cu}$. The numerical values obtained for $h_{N}$ from MD simulations are computed by averaging the different GB position steps in Figs. 7b and 8b. The comparisons between these values and the theoretical ones given by Eq. 53 are provided in Table 2 and show a very good agreement.

\begin{tabular}{cccc}
\hline$h_{N}(\mathrm{~nm})$ for $\langle 110\rangle$ mode & $\Sigma 41(540)$ & $\Sigma 5(210)$ & $\Sigma 17(410)$ \\
\hline Atomistic simulations (average values) & -0.255 & -0.248 & -0.220 \\
\hline Theory & -0.254 & -0.242 & -0.219
\end{tabular}

Table 2

Normal step height $h_{N}$ average values obtained by Eq. 53 (theory) compared to MD results for $\mathrm{Cu} \Sigma 41(540), \Sigma 5(210), \Sigma 17(410)$ [001] STGB.

Here, the shear-coupled GB migration event is assumed instantaneous at the continuum mechanics time scale so that during the stepwise GB motion $\Delta E_{i j}=\dot{E}_{i j} \delta t=0$ in Eq. 52, thus

$$
\Delta \Sigma_{i j}=\frac{h_{N}}{L}\left(C_{i j k l}^{e f f}\left[\varepsilon_{k l}\right]-\left[\sigma_{i j}\right]\right)
$$

Assuming linear isotropic homogeneous elasticity, Eq. 54 simplifies into

$$
\Delta \Sigma_{i j}=-2 \mu \frac{h_{N}}{L} \widetilde{R_{i j}} \beta
$$

since $\widetilde{R_{k k}}=0$ (see Subsection 3.3).

In the following, the tensor to matrix convention introduced in Appendix A is used. The pairs of subscripts $i j$ and $k l$ are converted to single subscripts as follows: $11 \rightarrow 1$, $22 \rightarrow 2,33 \rightarrow 3,23$ and $32 \rightarrow 4,13$ and $31 \rightarrow 5,12$ and $21 \rightarrow 6$. For simple shear 
parallel to GB plane in the $\left(\mathrm{x}_{1}\right)$ direction as performed in the MD simulations, and assuming a pure shear stress state over the bicrystal, the overall shear stress increment is obtained from Eq. 54 as follows

$$
\Delta \Sigma_{6}=\frac{h_{N}}{L}\left(C_{62}^{e f f}\left[\varepsilon_{2}\right]+C_{64}^{e f f}\left[\varepsilon_{4}\right]+C_{66}^{e f f}\left[\varepsilon_{6}\right]\right)
$$

since $\left[\sigma_{6}\right]=0$ (see Eq. A.2 in Appendix A). Here, the strain concentration equations (Eq. A.3 in Appendix A) are applied (shear strain-controlled problem). For isotropic elasticity, Eq. 55 simply yields

$$
\Delta \Sigma_{6}=-\mu \frac{h_{N}}{L} \widetilde{R_{6}} \beta
$$

with $\widetilde{R_{6}}=\cos \left(\frac{\pi}{2}-\theta\right)$. The overall effective plastic strain increment is obtained from Eq. 46 (using $\left[\sigma_{A}\right]=0$ ) and computed as follows

$$
\Delta E_{6}^{p e f f}=\Delta E_{6}^{p}=-\frac{h_{N}}{L}\left(\left[\varepsilon_{6}\right]-S_{61}^{e f f}\left[\sigma_{1}\right]-S_{63}^{e f f}\left[\sigma_{3}\right]-S_{65}^{e f f}\left[\sigma_{5}\right]\right)
$$

For isotropic elasticity, Eq. 57 yields the overall plastic strain increment

$$
\Delta E_{6}^{p}=\frac{h_{N}}{L} \widetilde{R_{6}} \beta
$$

The present micromechanics-based model is able to describe the shear stress drop magnitude in the stick-slip behaviour (Fig. 11). The shear stress drop magnitude can be defined as the absolute value of the shear stress increment $\left|\Delta \Sigma_{6}\right|$ obtained from Eq. 56 or Eq. 57. The stick-slip behaviour is dependent on the grain boundary character (through $\theta$ ), the effective elastic properties of the bicrystal, the lattice parameter (through $h_{N}$ ) and the bicrystal finite size $L$. The numerical values regarding shear stress drop magnitudes for $\Sigma 41(540)\left(\theta=77.32^{\circ}\right), \Sigma 5(210)\left(\theta=53.13^{\circ}\right)$, $\Sigma 17(410)\left(\theta=28.07^{\circ}\right)$ are reported in Table 3 both from atomistic results and from the micromechanical approach (either Eq. 56 for anisotropic elasticity or Eq. 57 for isotropic elasticity). The $\beta_{<110>}$ coupling factors are respectively $-0.222,-0.667,-$ 1.200 for $\Sigma 41(540), \Sigma 5(210), \Sigma 17(410)$. The quantitative comparisons reported in 
Table 3 give reasonable agreement with the atomistic results in the case of anisotropic elasticity for the three investigated STGB. The relative errors with respect to MD results on overall shear stress stress drops are respectively $\sim 27 \%, \sim 44 \%, \sim 5$ $\%$ for $\Sigma 41(540), \Sigma 5(210), \Sigma 17(410)$. Conversely, the isotropic elasticity assumption give sometimes unrealistic results which are far from the MD results especially for $\Sigma 41(540)$ STGB where the relative error reaches $116 \%$. The results show that the isotropic elastic assumption may only be relevant for the particular case of $\Sigma 5(210)$ STGB.

Another example where the micromechanical approach can be applied is the spe-

\begin{tabular}{cccc}
\hline$\left|\Delta \Sigma_{6}\right|(\mathrm{MPa})$ for $L=12.2 \mathrm{~nm}$ & $\Sigma 41(540)$ & $\Sigma 5(210)$ & $\Sigma 17(410)$ \\
\hline Atomistic simulations & $100(0 \mathrm{~K}, 500 \mathrm{~K})$ & $417(0 \mathrm{~K}, 500 \mathrm{~K})$ & $1080(0 \mathrm{~K})$ \\
\hline Micromechanical model (anisotropic) & 127 & 603 & 1024 \\
\hline Micromechanical model (isotropic) & 216 & 506 & 485 \\
\hline
\end{tabular}

Shear stress drop magnitudes (in $\mathrm{MPa}$ ) obtained by the MD results and by the micromechanical model with anisotropic and isotropic elastic formulations for $\mathrm{Cu} \Sigma 41(540)$, $\Sigma 5(210), \Sigma 17(410)$ [001] STGB with $L=12.2 \mathrm{~nm}$.

cific $\mathrm{Cu} \Sigma 13(320)\left(\theta=67.4^{\circ}\right)$ STGB investigated by Mishin et al. (2007) for which $\beta=-0.4$. In this case, the shear stress drop magnitude obtained by the authors using atomistic simulations at $0 \mathrm{~K}$ with $L=6 \mathrm{~nm}$ was found to be $\sim 450 \mathrm{MPa}$ (Mishin et al., 2007). Taking into account anisotropic elasticity (Eq. 56) and the fact that the "mode II" is active at $0 \mathrm{~K}$ (Eq. 53), the present model gives $\left|\Delta \Sigma_{6}\right|=573 \mathrm{MPa}$, which represents a relative error of $\sim 27 \%$, while isotropic elasticity would give $\left|\Delta \Sigma_{6}\right|=736 \mathrm{MPa}$ (relative error of $\sim 63 \%$ ).

For $\mathrm{Cu} \Sigma 17$ (410) STGB, "mode I" occurs at 500K according to Fig. 11c. In 
this $<100>$ mode, $B_{i}$ (or $l_{i}$ ) forms an angle $\theta / 2$ counterclockwise with respect to $n_{i}$ (Fig. 4) such that $l_{i} n_{i}=g_{i} t_{i}=\cos \left(\frac{\theta}{2}\right), l_{i}=\left(-\sin \left(\frac{\theta}{2}\right), \cos \left(\frac{\theta}{2}\right), 0\right), \mathrm{g}_{i}=$ $\left(\cos \left(\frac{\theta}{2}\right), \sin \left(\frac{\theta}{2}\right), 0\right)$. Here, crystal II is the parent grain and only crystal I undergoes plastic deformation, thus $\left[\varepsilon_{i j}^{p}\right]=-\varepsilon_{i j}^{p \mathrm{I}}=-\widetilde{R_{i j}} \beta$ where $\widetilde{R_{i j}}$ is given by Eq. 39 . In this case, the first shear stress drop obtaind by MD simulations is $\sim 300 \mathrm{MPa}$. The micromechanical model can also be applied in the same way to the "mode I" observed at $\mathrm{T}=500 \mathrm{~K}$. In this case, the theoretical $h_{N}$ value is now positive and is given by (Cahn et al., 2006a)

$$
h_{N}<100>=\frac{a_{L}}{2} \cos \left(\frac{\theta}{2}\right)
$$

Using Eq. 56 (elastic anisotropy), we obtain $\left|\Delta \Sigma_{6}\right|=322 \mathrm{MPa}$, which represents a relative error less than $8 \%$.

The continuum-based model is also able to describe the dissipated energy during shear-coupled GB migration (Fig. 12). The methodology to compute the dissipated energy during the first GB migration event is described by the grey area in Fig. 13 and is applied to the three STGB in comparison with MD simulations performed at $0 \mathrm{~K}$ for $L=12.2 \mathrm{~nm}$. From the knowledge of the critical shear stress denoted $\Sigma_{6}^{c}$ in Fig. 13 (left) which is obtained from MD simulations, the grey area (dissipated energy) is computed with the model by the product $\Sigma_{6}^{\star} \Delta E_{6}^{p}$ (see Fig. 13 (right)) with $\Sigma_{6}^{\star}=\Sigma_{6}^{c}+\Delta \Sigma_{6} / 2 . \Delta E_{6}^{p}$ and $\Delta \Sigma_{6}$ are respectively obtained from Eqs. 58 and 56 in the case of anisotropic elasticity and from Eqs. 59 and 57 in the case of isotropic elasticity. The quantitative comparisons between the dissipated energy using the previous methodology and the grey area directly obtained under the stressstrain curve given by MD simulations at 0K (see Fig. 7) are reported in Table 4. These results confirm a reasonable agreement with the atomistic results in the case of anisotropic elasticity for the three investigated STGB. In this case, the relative errors with respect to MD results on dissipated energy are respectively $\sim 14 \%, \sim$ 
$23 \%, \sim 6 \%$ for $\Sigma 41(540), \Sigma 5(210), \Sigma 17(410)$.
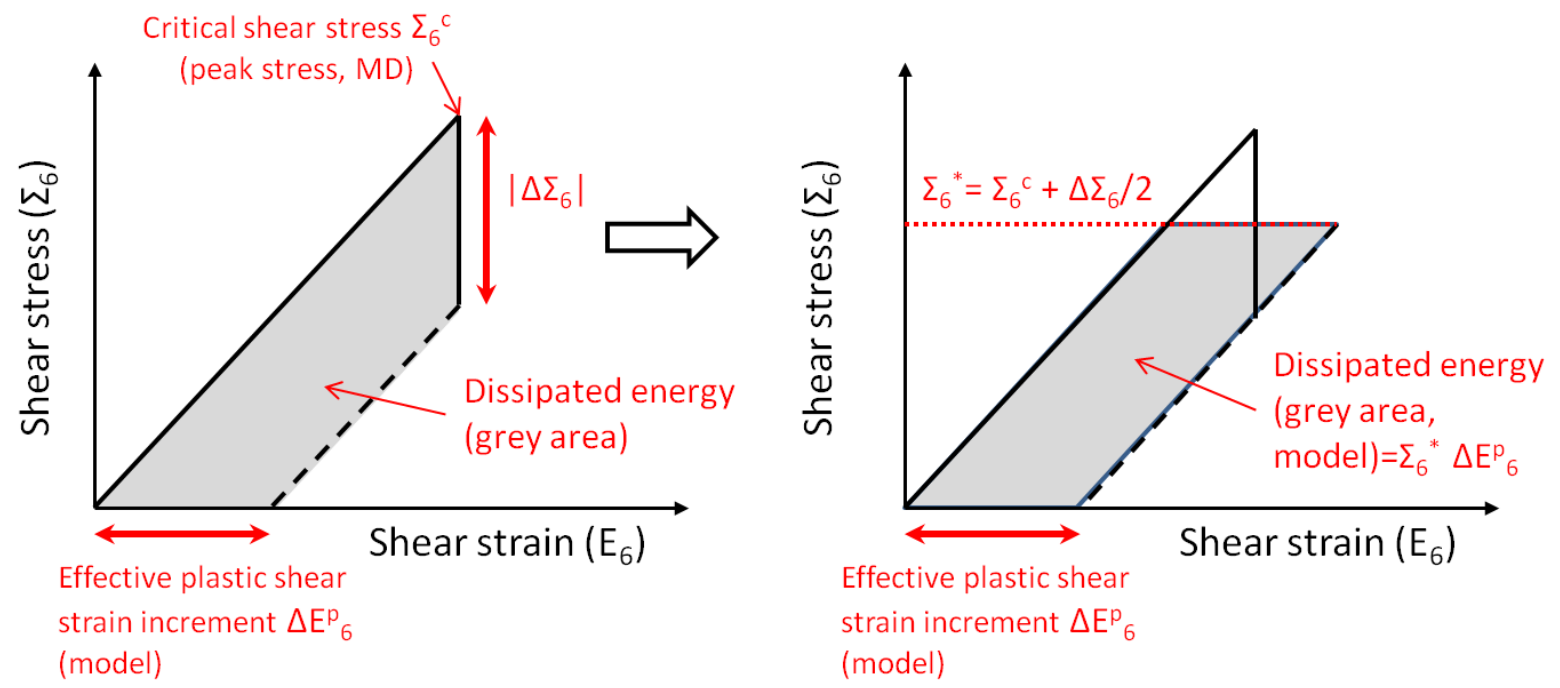

Figure 13. Schematic representation of the dissipated energy during first shear-coupled GB migration event (left), method to compute the dissipated energy with the micromechanical model and the knowledge of $\Sigma_{6}^{c}$ (right). 
Dissipated energy $\left(M J / m^{3}\right)$ for $L=12.2 \mathrm{~nm} \quad \Sigma 41(540) \quad \Sigma 5(210) \quad \Sigma 17(410)$

\begin{tabular}{cccc}
\hline Atomistic simulations (0K) & 5.31 & 12.70 & 39.90 \\
\hline Micromechanical model (anisotropic) & 6.04 & 15.60 & 37.55 \\
\hline Micromechanical model (isotropic) & 10.26 & 15.08 & 18.59 \\
\hline
\end{tabular}

Table 4

Dissipated energy (in $M J / m^{3}$ ) obtained by the MD results and by the micromechanical model with anisotropic and isotropic elastic formulations for $\mathrm{Cu} \Sigma 41(540), \Sigma 5(210)$, $\Sigma 17(410)$ [001] STGB with $L=12.2 \mathrm{~nm}$ at $0 \mathrm{~K}$.

\subsubsection{Prediction of bicrystal size effects on shear stress drop}

Regarding the bicrystal size effect investigated in Subsection 4.2 for $\Sigma 41(540), \Sigma 5(210)$, $\Sigma 17(410)$ on shear stress drop magnitude, the results of the micromechanical model for $L=20 \mathrm{~nm}$ are given in Table 4 (for both isotropic and anisotropic formulations). Overall, the bicrystal size effect is well reproduced for both STGB in the case where anisotropic elasticity is accounted for, especially for $\Sigma 41(540)$ and $\Sigma 17(410)$. Even though the micromechanical approach supposes mean strain and stress fields in both crystals (no intracrystalline shear stress fluctuations along the normal axis $\left(\mathrm{x}_{2}\right)$ to the GB plane, see Eq. A.2), it is found that the bicrystal size effect (characterized by the internal length scale $L$ ) on the shear stress drop magnitude scales with $\frac{h_{N}}{L}$. Here, $h_{N}$ is fixed for a given STGB because it is linked to the lattice parameter $a_{L}$ in Eq. 53. Following Eq. 56 and the fact that the terms $C_{62}^{e f f}\left[\varepsilon_{2}\right]+C_{64}^{e f f}\left[\varepsilon_{4}\right]+C_{66}^{e f f}\left[\varepsilon_{6}\right]$ are not length scale dependent (see Appendix A), the stress drop magnitude ratio $\frac{\left|\Delta \Sigma_{6}\right|_{L=12.2 \mathrm{~nm}}}{\left|\Delta \Sigma_{6}\right|_{L=20 \mathrm{~nm}}}=\frac{20}{12.2} \sim 1.64$ is in good agreement with the one obtained by atomistic simulations for $\Sigma 41(540), \Sigma 5(210)$ and $\Sigma 17(410)$ which are respectively $1.67,1.71$ and 1.68 from Tables 3 and 5 . 


\begin{tabular}{cccc}
\hline$\left|\Delta \Sigma_{6}\right|(\mathrm{MPa})$ for $L=20 \mathrm{~nm}$ & $\Sigma 41(540)$ & $\Sigma 5(210)$ & $\Sigma 17(410)$ \\
\hline Atomistic simulations (0K) & 60 & 244 & 641 \\
\hline Micromechanical model (anisotropic) & 78 & 368 & 624 \\
\hline Micromechanical model (isotropic) & 132 & 309 & 296 \\
\hline
\end{tabular}

Table 5

Shear stress drop magnitudes (in $\mathrm{MPa}$ ) obtained by the MD results and by the micromechanical model with anisotropic and isotropic elastic formulations for $\mathrm{Cu} \Sigma 41(540)$, $\Sigma 5(210), \Sigma 17(410)[001]$ STGB with $L=20 \mathrm{~nm}$ at $0 \mathrm{~K}$.

\subsection{Discussion}

It looks clear that the large discrepancies sometimes observed between the atomistic results and the micromechanical model with the isotropic elastic assumption are mainly due to a poor estimate of the effective elastic shear modulus and the strains in both crystals. The predictions of shear stress drops and dissipated energy during first shear-coupling GB migration are in reasonable agreement with MD results when anisotropic elasticity is considered in the formulation. Furthermore, it has been checked that atomistic simulations give approximately same averaged shear stresses in crystals I and II which is here consistent with the continuum stress equilibrium requirement (see Eq. A.2). In the continuum model, a small strain formulation is used with only linear elastic effects. No normal applied stress is considered for boundary conditions. It is assumed that the overall stresses of the bicrystal are in pure shear stress under controlled shear strain for the calculations of stresses and strains in each crystal during shear-coupled GB migration. According to Fig. 14, MD simulations show the presence of overall normal stress (i.e. the averaged stress over all dynamic 
atoms along the normal direction to the GB plane) in addition to overall shear stress. This normal stress nonlinearly increases with applied shear and the normal stress variation at the occurrence of shear-coupled GB migration reaches 130MPa. These second order non linear effects (with respect to first order linear anisotropic elasticity effects) might contribute to the differences observed between the continuum-based model and MD results. Geometrical and material nonlinearities (see e.g Wu (2005)) are not considered in the present micromechanical model and are left for further studies.

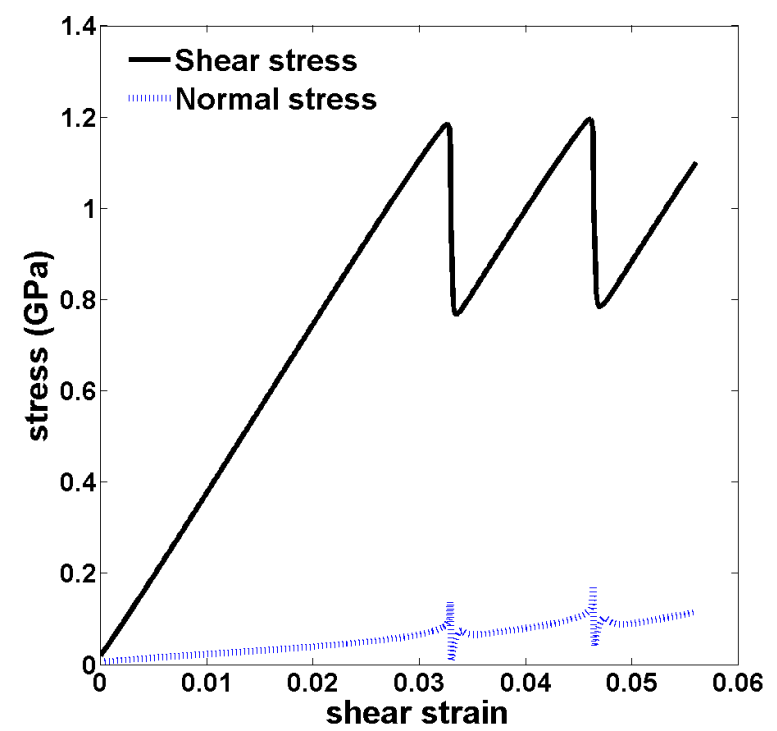

Figure 14. Overall shear stress (solid line) and normal stress (dotted line) computed by MD simulations for $\Sigma 5(210)$ at $0 \mathrm{~K}(L=12.2 \mathrm{~nm})$ during applied simple shear deformation.

The results of the present study show that as grains grow due to shear-coupled GB migration, it is seen that both GB character and elastic anisotropy will produce elastic and plastic incompatibilities under shear deformation which can affect the kinetics and morphology of grains during plastic deformation. In real polycrystalline metals, GB mobility is very difficult to capture because of the presence of different GB characters. An arbitrary mixed GB boundary with tilt and twist components or asymmetric GB (Zhang et al., 2008) could also be considered by the present 
continuum-based approach. Inspecting the general expression for the driving force $F_{f}$ for normal GB motion in Eq. 41, it is found that for elastically anisotropic crystals (like $\mathrm{Cu}$ ), the shear stress can create a volume driving force due to the difference between the elastic strain energy densities in the grains. Conversely, shear-coupled GB migration may also be forbidden for specific situations which cancel $F_{f}$. In polycrystals, GBs have generally no pure tilt or pure twist characters. However, it was recently observed (Morawiec, 2009) for polycrystals with cubic symmetry that more than $80 \%$ of random GB are near tilt GB and less than $5 \%$ are near twist ones. The mechanical framework developed in this paper holds for both low angle and high angle GBs. It is also thought that extended kinematics for shear-coupled GB migration (Mompiou et al., 2010, 2011) could also be incorporated in this framework and merits further studies. Twin boundaries are special cases where the present model can also be applied (twinning does not involve diffusion) by considering the characteristic twinning shear as input of the model. In this particular case, the continuum model can also be applied without preliminary atomistic studies to identify shear modes and integrated in recent polycrystalline models (Shiekhelsouk et al., 2009). As another application of the present constitutive model, stress-induced GB migration can produce grain shape changes during plastic deformation of nanocryscrystalline materials. Today, it is important to well handle in-use mechanical properties of nanocrystalline materials by a good compromise between yield strength and ductility. These materials exhibit a strong yield stress (due to the Hall-Petch's behavior even though this one is seen to be reversed at very fine grain sizes) but for most of them ductility appears to be poor (Dao et al., 2007). Shear-coupled GB migration is another deformation mechanism in competition to diffusional creep, dislocation nucleation and slip, which is characterized by a different grain-size and temperature dependencies of the strain rate. This mechanism should be especially important in the study of the deformation behaviour of nanocrystalline materials where grain size 
distribution (Berbenni et al., 2007; Nicaise et al., 2011) and GB mechanisms (Barai and Weng, 2009; Schillebeeckx et al., 2011) play a key role on their overall behaviour. Due to the high resolved shear stresses required to move STGB, it is expected that GB migration would play a key role in the understanding of inverse Hall-Petch effect in addition to GB sliding or GB dislocation nucleation-propagation-absorption. Furthermore, it is important to well model the inelastic accommodation mechanisms at grain boundaries and junctions in order to understand the initiation of cracks in these materials (Bobylev et al., 2010).

\section{Conclusions}

A new micromechanics-based model was investigated to describe shear-coupled GB migration in bicrystals. Both MD simulations (at $0 \mathrm{~K}$ and $500 \mathrm{~K}$ ) and a micromechanical model assuming Frank-Bilby GB dislocations were applied to three $\mathrm{Cu}[001]$ symmetric tilt GBs: $\Sigma 41(540)\left(\theta=77.32^{\circ}\right), \Sigma 5(210)\left(\theta=53.13^{\circ}\right), \Sigma 17(410)\left(\theta=28.07^{\circ}\right)$. The critical shear stresses (or peak stresses) for shear-coupled GB migration have been obtained by MD simulations under fixed temperatures. The role of copper elastic anisotropy on the stick-slip features of shear-coupled migration has been observed on the shear stress-strain curves. These ones have been analyzed in the light of the micromechanical model.

The $\beta$ geometric parameter (the so-called shear coupling factor) used in materials science) is derived from the Hadamard's kinematics relation for particle velocity jump at the interface. This is also consistent with the kinematics of GB migration studied at the atomistic level (Tucker et al., 2011). This shows the efficiency of the continuum-based approach even at the nanoscale. The driving forces for shear-coupled migration can be developed using either isotropic elasticity or cubic 
(anisotropic) elasticity. The formulation is consistent with the concept of configurational forces and the Eshelby energy-momentum based thermo-micromechanics. The effective elastic moduli of the bicrystals computed with anisotropic elasticity well match the atomistic results using the EAM potential for copper from Mishin et al. (2001). The jumps of plastic strains and plastic distortions at the interface (eigenstrains and eigendistortions) are derived and depend on the $\beta$ geometric parameter and an orientation tensor for GB dislocation slip. The effective plastic strain rate of the bicrystal during a GB migration event is computed and depends on the strain and stress jumps at the interface. This formula which is based on transport equations is quite general and can be applied to other GB without any difficulty.

It is found that the predictions of shear stress drops and dissipated energy during first shear-coupling GB migration event at fixed temperatures are well described by the micromechanical approach especially when anisotropic elasticity is considered in the formulation. The model may also be extended to various strain rates and temperatures assuming the shear-coupling modes can be easily identified by atomistic simulations. Interestingly, for very low velocities up to $5 \mathrm{~m} / \mathrm{s}$, the shear stress drop is not very sensitive to shear rates (Mishin et al., 2007). Advanced atomistic methods dedicated to obtain the kinetics parameters of shear-coupled GB migration should be developed to improve the constitutive kinetics law and to obtain the GB mobilities at finite temperatures. Real experimental data for $\mathrm{Cu}$ STGB would be also ideal to validate the model in addition to atomistic simulations. Nevertheless, experiments to observe the stick-slip behavior should consider not too large grain size (i.e. preferably in the nanoscale range) which needs in situ high-resolution electron microscopy.

As some perspective to polycrystal modeling and applications, the present work developed for bicrystals with shear-coupled GB migration can be applied to study 
stress-induced twin boundary migration and nanocrystalline materials as discussed previously. The constitutive framework will be imported in polycrystalline models like the ones recently developed by the authors (Berbenni et al., 2007; Shiekhelsouk et al., 2009; Nicaise et al., 2011) by considering GB character dependent behaviour in addition to crystals behaviours.

\section{Acknowledgements}

$\mathrm{SB}$ and $\mathrm{MC}$ are grateful for the support of the french "Agence Nationale de la Recherche" under contract "Nanocrystals" (ANR-07-BLAN-0186). 


\section{Appendix}

\section{A Stress and strain concentration equations, effective elastic behaviour for planar GB with general heterogeneous elasticity}

Here, a "representative" volume $V$ as the one represented in Fig. 2 is considered where crystal I occupies a volume fraction $f=V_{I} / V$ and crystal II occupies a volume fraction 1-f. In order to find the "effective" elastic moduli of the bicrystal with planar grain boundaries, different techniques can be applied like the one described in Stupkiewicz and Petryk (2002),using in-plane and out-of-plane components for stresses and strains, or the one described in Franciosi and Berbenni (2007, 2008) using the "Transformation Field Analysis" (TFA) for two-phase material introduced in the nineties by Dvorak (1990). In this paper, we will avoid the calculations of the TFA influence tensors from the elastic concentration tensors, and we will apply the framework given in Stupkiewicz and Petryk (2002) to a general elastic anisotropic bicrystals constituted of crystals I and II (Fig A.1). Here, we give the explicit expressions for general anisotropic elasticity in both crystals after setting the strain (resp. stress) concentration equations and the effective elastic moduli (resp. compliances). It is noteworthy that only the stress concentration equations were reported in Stupkiewicz and Petryk (2002) (not the strain concentration equations as detailed and applied in the present paper). The linear elastic behaviour of a crystal with plastic strains (or eigenstrains) denoted $\varepsilon_{i j}^{p}$ is governed by the elastic Hooke's law in the form $\sigma_{i j}=C_{i j k l}\left(\varepsilon_{k l}-\varepsilon_{k l}^{p}\right)$ or its inverse relationship $\varepsilon_{i j}=S_{i j k l} \sigma_{k l}+\varepsilon_{i j}^{p}$, where $C_{i j k l}$ and $S_{i j k l}$ are respectively the components of the elastic moduli and compliance tensors. Here, we adopt the following tensor to matrix convention (Nye, 1957) in which pairs of subscripts ij and $k l$ are converted to single subscripts: $11 \rightarrow 1,22 \rightarrow 2,33 \rightarrow 3,23$ and $32 \rightarrow 4,13$ 
and $31 \rightarrow 5,12$ and $21 \rightarrow 6$. Thus, each of the tensor components is associated with a matrix component as follows

$$
\begin{aligned}
& \left(\begin{array}{rrr}
\sigma_{11} & \sigma_{12} & \sigma_{13} \\
\sigma_{22} & \sigma_{23} \\
& \\
& \sigma_{33}
\end{array}\right) \rightarrow\left(\begin{array}{rrr}
\sigma_{1} & \sigma_{6} & \sigma_{5} \\
& \\
\sigma_{2} & \sigma_{4} \\
& \\
& \sigma_{3}
\end{array}\right) \\
& \left(\begin{array}{ccc}
\varepsilon_{11} & \varepsilon_{12} & \varepsilon_{13} \\
& \varepsilon_{22} & \varepsilon_{23} \\
& & \\
& & \varepsilon_{33}
\end{array}\right) \rightarrow\left(\begin{array}{rrrr}
\varepsilon_{1} & \frac{1}{2} \varepsilon_{6} & \frac{1}{2} \varepsilon_{5} \\
& \varepsilon_{2} & \frac{1}{2} \varepsilon_{4} \\
& & \\
& & \varepsilon_{3}
\end{array}\right) \\
& C_{i j k l} \rightarrow C_{m n} \\
& S_{i j k l} \rightarrow\left\{\begin{array}{c}
S_{m n} \text { if } \mathrm{m}, \mathrm{n}=1,2,3 \\
\frac{S_{m n}}{2} \text { if either } \mathrm{m} \text { or } \mathrm{n}=4,5,6 \\
\frac{S_{m n}}{4} \text { if both } \mathrm{m} \text { and } \mathrm{n}=4,5,6
\end{array}\right.
\end{aligned}
$$

The Hooke's law writes $\sigma_{m}=C_{m n}\left(\varepsilon_{n}-\varepsilon_{n}^{p}\right)$ and $\varepsilon_{m}=S_{m n} \sigma_{n}+\varepsilon_{m}^{p}$ for $\mathrm{m}, \mathrm{n}=1,2, \ldots 6$ where $C_{m n}, S_{m n}$ are respectively the $6 \times 6$ square matrix forms of elastic moduli and compliances. Considering the bicrystal's configuration in Fig. A.1, the in-plane matrix components are denoted $\mathrm{P}=1,3,5$, and the out of plane (or anti-plane) components are denoted $\mathrm{A}=2,4,6$. The application of both traction vector continuity and tangential strain continuity (Hadamard, 1903) yields

$$
\begin{aligned}
& {\left[\sigma_{A}\right]=0 \Leftrightarrow \sigma_{A}^{I}=\sigma_{A}^{I I}=\Sigma_{A}} \\
& {\left[\varepsilon_{P}\right]=0 \Leftrightarrow \varepsilon_{P}^{I}=\varepsilon_{P}^{I I}=E_{P}}
\end{aligned}
$$


where the averaging rules $\Sigma_{i j}=f \sigma_{i j}^{I}+(1-f) \sigma_{i j}^{I I}$ and $E_{i j}=f \varepsilon_{i j}^{I}+(1-f) \varepsilon_{i j}^{I I}$ apply.

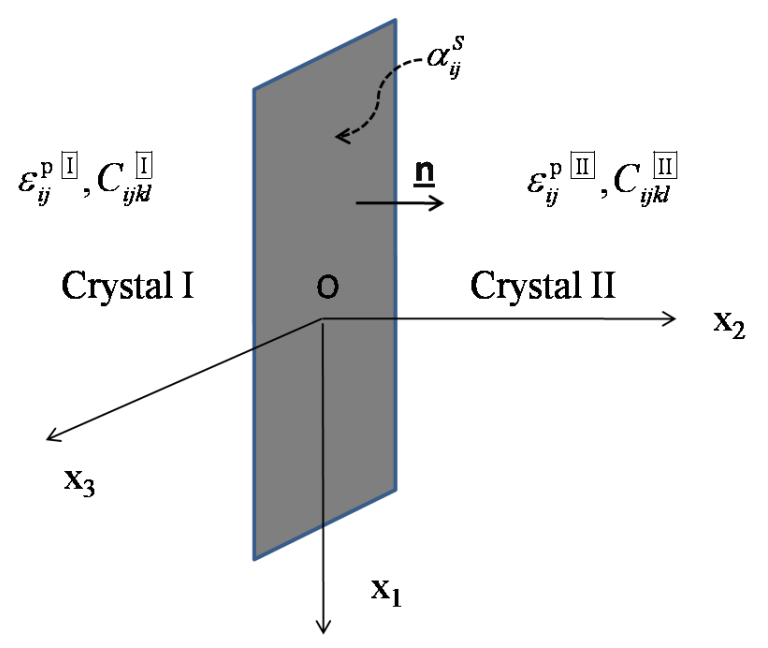

Figure A.1. Schematic representation of the bicrystal with the coordinate axes used to derive the tensor or matrix components throughout the paper.

After some algebraic manipulations, we find the strain concentration equations for the out of plane strain components as follows (with summations over repeated indices $\mathrm{A}=2,4,6$ and $\mathrm{P}=1,3,5)$

$$
\begin{aligned}
& \varepsilon_{A}^{I}=A_{A A}^{I} E_{A}+A_{A P}^{I} E_{P}-(1-f) G_{A A}\left[\sigma_{A}^{p}\right] \\
& \varepsilon_{A}^{I I}=A_{A A}^{I I} E_{A}+A_{A P}^{I I} E_{P}+f G_{A A}\left[\sigma_{A}^{p}\right]
\end{aligned}
$$


where $A_{A A}^{I}, A_{A A}^{I I}, A_{A P}^{I}, A_{A P}^{I I}$ (elastic strain concentration components), $G_{A A}$ and $\left[\sigma_{A}^{p}\right]$ are given as follows

$$
\begin{aligned}
& A_{A A}^{I}=\left(C_{A A}^{*}\right)^{-1} C_{A A}^{I I} \\
& A_{A P}^{I}=(1-f)\left(C_{A A}^{*}\right)^{-1}\left[C_{A P}\right] \\
& A_{A A}^{I I}=\left(C_{A A}^{*}\right)^{-1} C_{A A}^{I} \\
& A_{A P}^{I I}=-f\left(C_{A A}^{*}\right)^{-1}\left[C_{A P}\right] \\
& G_{A A}=\left(C_{A A}^{*}\right)^{-1} \\
& {\left[\sigma_{A}^{p}\right]=C_{A A}^{I I} \varepsilon_{A}^{p I I}+C_{A P}^{I I} \varepsilon_{P}^{p I I}-C_{A A}^{I} \varepsilon_{A}^{p I}-C_{A P}^{I} \varepsilon_{P}^{p I}}
\end{aligned}
$$

with $C_{A A}^{*}=(1-f) C_{A A}^{I}+f C_{A A}^{I I}$. According to Eqs. A.2 (second equation) and A.3, the elastic strain concentration tensors $A_{i j k l}^{I}, A_{i j k l}^{I I}$ and $G_{i j k l}$ can be recast as

$$
\begin{aligned}
A_{i j k l}^{I, I I} & \equiv\left(\begin{array}{cc}
A_{A A}^{I, I I} & A_{A P}^{I, I I} \\
0 & I
\end{array}\right) \\
G_{i j k l} & \equiv\left(\begin{array}{cc}
G_{A A} & 0 \\
0 & 0
\end{array}\right)
\end{aligned}
$$

By setting $\left[\sigma_{A}^{p}\right]=0$ (no plastic strain) in Eq. A.3 and using the static averaging rules, we find the effective elastic moduli $C_{i j k l}^{e f f}$ as follows

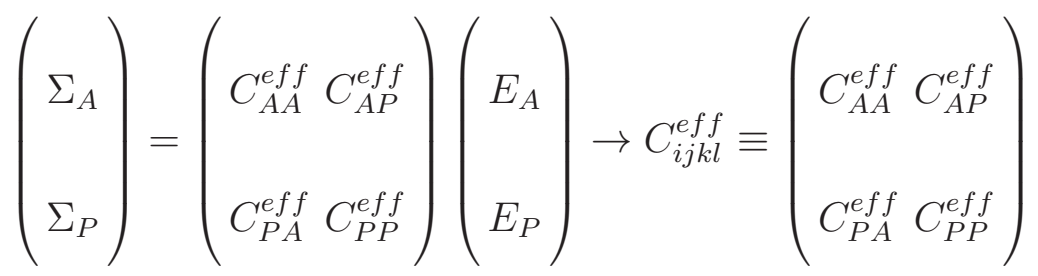

From Eq. A.6, the expressions of $C_{A A}^{e f f}, C_{A P}^{e f f}, C_{P A}^{e f f}, C_{P P}^{e f f}$ are obtained after algebraic manipulations using the elastic Hooke's laws for crystals I and II and 
Eqs. A.2-A.5

$$
\begin{aligned}
& C_{A A}^{e f f}=f C_{A A}^{I}\left(C_{A A}^{*}\right)^{-1} C_{A A}^{I I}+(1-f) C_{A A}^{I I}\left(C_{A A}^{*}\right)^{-1} C_{A A}^{I} \\
& C_{P A}^{e f f}=f C_{P A}^{I}\left(C_{A A}^{*}\right)^{-1} C_{A A}^{I I}+(1-f) C_{P A}^{I I}\left(C_{A A}^{*}\right)^{-1} C_{A A}^{I} \\
& C_{P P}^{e f f}=f C_{P P}^{I}+(1-f) C_{P P}^{I I}-f(1-f)\left(C_{P A}^{I I}-C_{P A}^{I}\right)\left(C_{A A}^{*}\right)^{-1}\left(C_{A P}^{I I}-C_{A P}^{I}\right) \\
& C_{A P}^{e f f}=f C_{A P}^{I}+(1-f) C_{A P}^{I I}-f(1-f)\left(C_{A A}^{I I}-C_{A A}^{I}\right)\left(C_{A A}^{*}\right)^{-1}\left(C_{A P}^{I I}-C_{A P}^{I}\right)
\end{aligned}
$$

The relations in Eq. A.7 are consistent with the expressions given in Stupkiewicz and Petryk (2002) (note that $C_{A A}^{e f f}$ and $C_{P A}^{e f f}$ are here written in a simpler form). Let us now focus on stress concentration equations. In addition to Eq. A.2 (first equation), we can directly follow the same authors to obtain the in plane stresses in both crystals

$$
\begin{aligned}
\sigma_{P}^{I} & =B_{P P}^{I} \Sigma_{P}+B_{P A}^{I} \Sigma_{A}+(1-f) F_{P P}\left[\varepsilon_{P}^{p}\right] \\
\sigma_{P}^{I I} & =B_{P P}^{I I} \Sigma_{P}+B_{P A}^{I I} \Sigma_{A}-f F_{P P}\left[\varepsilon_{P}^{p}\right]
\end{aligned}
$$

where $B_{P P}^{I}, B_{P P}^{I I}, B_{P A}^{I}, B_{P A}^{I I}$ (elastic stress concentration components), $F_{P P}$ and $\left[\varepsilon_{P}^{p}\right]$ are given by

$$
\begin{aligned}
& B_{P P}^{I}=\left(S_{P P}^{*}\right)^{-1} S_{P P}^{I I} \\
& B_{P A}^{I I}=(1-f)\left(S_{P P}^{*}\right)^{-1}\left[S_{P A}\right] \\
& B_{P P}^{I I}=\left(S_{P P}^{*}\right)^{-1} S_{P P}^{I} \\
& B_{P A}^{I I}=-f\left(S_{P P}^{*}\right)^{-1}\left[S_{P A}\right] \\
& F_{P P}=\left(S_{P P}^{*}\right)^{-1} \\
& {\left[\varepsilon_{P}^{p}\right]=\varepsilon_{P}^{p I I}-\varepsilon_{P}^{p I}}
\end{aligned}
$$


with $S_{P P}^{*}=(1-f) S_{P P}^{I}+f S_{P P}^{I I}$. Following Eq. A.2 (first equation) and Eq. A.8, the elastic stress concentration tensors $B_{i j k l}^{I}, B_{i j k l}^{I I}$ and $F_{i j k l}$ can recast as

$$
\begin{aligned}
& B_{i j k l}^{I, I I} \equiv\left(\begin{array}{cc}
I & 0 \\
B_{P A}^{I, I I} & B_{P P}^{I, I I}
\end{array}\right) \\
& F_{i j k l} \equiv\left(\begin{array}{cc}
0 & 0 \\
0 & F_{P P}
\end{array}\right)
\end{aligned}
$$

By setting $\left[\varepsilon_{P}^{p}\right]=0$ (no plastic strain) in Eq. A.8 and using the averaging rules, we find the effective elastic compliances $S_{i j k l}^{e f f}$ as follows

$$
\left(\begin{array}{c}
E_{A} \\
E_{P}
\end{array}\right)=\left(\begin{array}{cc}
S_{A A}^{e f f} & S_{A P}^{e f f} \\
& \\
S_{P A}^{e f f} & S_{P P}^{e f f}
\end{array}\right)\left(\begin{array}{c}
\Sigma_{A} \\
\Sigma_{P}
\end{array}\right) \rightarrow S_{i j k l}^{\text {eff }} \equiv\left(\begin{array}{cc}
S_{A A}^{\text {eff }} & S_{A P}^{\text {eff }} \\
& \\
S_{P A}^{\text {eff }} & S_{P P}^{\text {eff }}
\end{array}\right)
$$

From Eq. A.11, the expressions of $S_{P P}^{e f f}, S_{A P}^{e f f}, S_{P A}^{e f f}, S_{A A}^{e f f}$ are obtained after algebraic manipulations using the elastic Hooke's laws for crystals I and II and Eqs. A.2, A.8-A.10

$$
\begin{aligned}
& S_{P P}^{e f f}=f S_{P P}^{I}\left(S_{P P}^{*}\right)^{-1} S_{P P}^{I I}+(1-f) S_{P P}^{I I}\left(S_{P P}^{*}\right)^{-1} S_{P P}^{I} \\
& S_{A P}^{e f f}=f S_{A P}^{I}\left(S_{P P}^{*}\right)^{-1} S_{P P}^{I I}+(1-f) S_{A P}^{I I}\left(S_{P P}^{*}\right)^{-1} S_{P P}^{I} \\
& S_{A A}^{e f f}=f S_{A A}^{I}+(1-f) S_{A A}^{I I}-f(1-f)\left(S_{A P}^{I I}-S_{A P}^{I}\right)\left(S_{P P}^{*}\right)^{-1}\left(S_{P A}^{I I}-S_{P A}^{I}\right) \\
& S_{P A}^{e f f}=f S_{P A}^{I}+(1-f) S_{P A}^{I I}-f(1-f)\left(S_{P P}^{I I}-S_{P P}^{I}\right)\left(S_{P P}^{*}\right)^{-1}\left(S_{P A}^{I I}-S_{P A}^{I}\right)
\end{aligned}
$$

The relations in Eq. A.12 are also consistent with the expressions given in Stupkiewicz and Petryk (2002). In order to determine $A_{i j k l}^{I}, A_{i j k l}^{I I}, G_{i j k l}, C_{i j k l}^{e f f}, B_{i j k l}^{I}$, $B_{i j k l}^{I I}, F_{i j k l}, S_{i j k l}^{e f f}$ in Eqs. A.4-A.12, the different matrix forms and are detailed in the particular configuration of Fig. A.1 as follows (the formulas remain valid for 
any other configuration with different axes using indices permutation)

$$
\begin{aligned}
& C_{A A}^{I, I I}=\left(\begin{array}{ccc}
C_{22}^{I, I I} & C_{24}^{I, I I} & C_{26}^{I, I I} \\
C_{42}^{I, I I} & C_{44}^{I, I I} & C_{46}^{I, I I} \\
C_{62}^{I, I I} & C_{64}^{I, I I} & C_{66}^{I, I I}
\end{array}\right) ; C_{A P}^{I, I I}=\left(\begin{array}{ccc}
C_{21}^{I, I I} & C_{23}^{I, I I} & C_{25}^{I, I I} \\
C_{41}^{I, I I} & C_{43}^{I, I I} & C_{45}^{I, I I} \\
C_{61}^{I, I I} & C_{63}^{I, I I} & C_{65}^{I, I I}
\end{array}\right)
\end{aligned}
$$

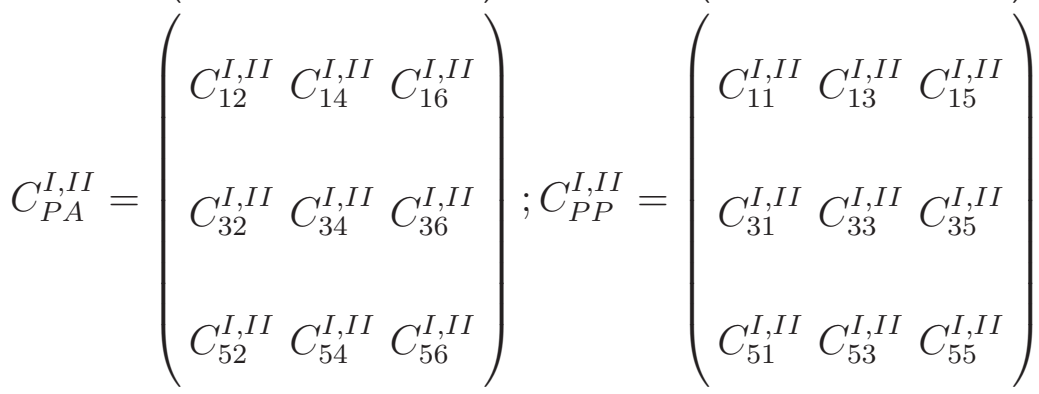

$$
C_{A A}^{*}=\left(\begin{array}{ccc}
C_{22}^{*} & C_{24}^{*} & C_{26}^{*} \\
C_{42}^{*} & C_{44}^{*} & C_{46}^{*} \\
C_{62}^{*} & C_{64}^{*} & C_{66}^{*}
\end{array}\right) \rightarrow G_{A A}=\left(C_{A A}^{*}\right)^{-1}
$$

$$
\begin{aligned}
S_{A A}^{I, I I}=\left(\begin{array}{ccc}
S_{22}^{I, I I} & S_{24}^{I, I I} & S_{26}^{I, I I} \\
& & \\
S_{42}^{I, I I} & S_{44}^{I, I I} & S_{46}^{I, I I} \\
S_{62}^{I, I I} & S_{64}^{I, I I} & S_{66}^{I, I I}
\end{array}\right) ; S_{A P}^{I, I I}=\left(\begin{array}{ccc}
S_{21}^{I, I I} & S_{23}^{I, I I} & S_{25}^{I, I I} \\
& & \\
S_{41}^{I, I I} & S_{43}^{I, I I} & S_{45}^{I, I I} \\
& & \\
S_{61}^{I, I I} & S_{63}^{I, I I} & S_{65}^{I, I I}
\end{array}\right) \\
S_{P A}^{I, I I}=\left(\begin{array}{llll}
S_{12}^{I, I I} & S_{14}^{I, I I} & S_{16}^{I, I I} \\
S_{32}^{I, I I} & S_{34}^{I, I I} & S_{36}^{I, I I} \\
S_{52}^{I, I I} & S_{54}^{I, I I} & S_{56}^{I, I I}
\end{array}\right) ; S_{P P}^{I, I I}=\left(\begin{array}{ccc}
S_{11}^{I, I I} & S_{13}^{I, I I} & S_{15}^{I, I I} \\
& & \\
S_{31}^{I, I I} & S_{33}^{I, I I} & S_{35}^{I, I I} \\
& & \\
S_{51}^{I, I I} & S_{53}^{I, I I} & S_{55}^{I, I I}
\end{array}\right)
\end{aligned}
$$




$$
S_{P P}^{*}=\left(\begin{array}{ccc}
S_{11}^{*} & S_{13}^{*} & S_{15}^{*} \\
S_{31}^{*} & S_{33}^{*} & S_{35}^{*} \\
S_{51}^{*} & S_{53}^{*} & S_{55}^{*}
\end{array}\right) \rightarrow F_{P P}=\left(S_{P P}^{*}\right)^{-1}
$$

For a given volume fraction $f$ of crystal I, the strain and stress jumps $\left[\varepsilon_{i j}\right],\left[\sigma_{i j}\right]$ and stress average $\left\langle\sigma_{i j}\right\rangle$ are easily obtained from Eqs. A.3 and A.8.

\section{B Application to cubic elasticity and bicrystals with [001] STGB}

In the reference cubic crystal with the cubic axes, there are only three independent elastic constants, namely $C_{11}, C_{12}, C_{44}$ such as in this frame $C_{i i i i}=C_{11}, C_{i i j j}=C_{12}, C_{i j i j}=C_{44}$ with $i \neq j$ (no summation over repeated indices). Then, for cubic symmetry, the elastic anisotropic constants in an arbitrary coordinate system are obtained by the following transformation rules (Dederichs and Leibfried, 1969; Gemperlova et al., 1989)

$$
\begin{aligned}
& C_{i j k l}=C_{12} \delta_{i j} \delta_{k l}+C_{44}\left(\delta_{i k} \delta_{j l}+\delta_{i l} \delta_{j k}\right)+C_{0} \sum_{s=1}^{3} e_{i}^{(s)} e_{j}^{(s)} e_{k}^{(s)} e_{l}^{(s)} \\
& S_{i j k l}=S_{12} \delta_{i j} \delta_{k l}+\frac{1}{4} S_{44}\left(\delta_{i k} \delta_{j l}+\delta_{i l} \delta_{j k}\right)+S_{0} \sum_{s=1}^{3} e_{i}^{(s)} e_{j}^{(s)} e_{k}^{(s)} e_{l}^{(s)}
\end{aligned}
$$

where $e_{i}^{(1)}, e_{i}^{(2)}, e_{i}^{(3)}$ are the unit vectors of the cubic lattice system ([100], [010], [001]), $C_{0}=C_{11}-C_{12}-2 C_{44}$ and $S_{0}=S_{11}-S_{12}-\frac{1}{2} S_{44}$. The elastic compliances and moduli are linked by

$$
\begin{aligned}
& S_{11}=\left(C_{11}+C_{12}\right) /\left\{\left(C_{11}-C_{12}\right)\left(C_{11}+2 C_{12}\right)\right\} \\
& S_{12}=-C_{12} /\left\{\left(C_{11}-C_{12}\right)\left(C_{11}+2 C_{12}\right)\right\} \\
& S_{44}=1 / C_{44}
\end{aligned}
$$

For bicrystals with [001] STGB, the cubic unit vector [001] is parallel to the $\left(\mathrm{x}_{1}\right)$ axis (tilt axis) (Fig. A.1). If we denote $\psi$ the rotation angle around the tilt axis of 
the cubit unit vector [100] with respect to the (x2) axis normal to the GB plane, then $\psi^{I}=-\frac{\theta}{2}$ and $\psi^{I I}=\frac{\theta}{2}$ such that $\theta=\psi^{I I}-\psi^{I}$ is the misorientation angle. In this configuration, the unit vectors of the cubic lattice system read

$$
e_{i}^{(1)}=\left(\begin{array}{c}
\cos \psi \\
\sin \psi \\
0
\end{array}\right), e_{i}^{(2)}=\left(\begin{array}{c}
-\sin \psi \\
\cos \psi \\
0
\end{array}\right), e_{i}^{(3)}=\left(\begin{array}{l}
0 \\
0 \\
1
\end{array}\right)
$$

Thus, Eqs. A.12-A.16 can be computed using Eqs. B.1-B.3.

\section{References}

Abeyaratne, R., Knowles, J. K., 1990. On the driving traction acting on a surface of strain discontinuity in a continuum. J. Mech. Phys. Solids 38, 345-360.

Acharya, A., 2001. A model of crystal plasticity based on the theory of continuously distributed dislocations. J. Mech. Phys. Solids 49, 761-785.

Barai, P., Weng, G. J., 2009. Mechanics of very fine-grained nanocrystalline materials with contributions from grain interior, GB zone, and grain-boundary sliding. Int. J. Plast. 25, 2410-2434.

Berbenni, S., Favier, V., Berveiller, M., 2007. Impact of the grain size distribution on the yield stress of heterogeneous materials. Int. J. Plast. 23, 114-142.

Bilby, B. A., 1955. Types of dislocation sources. In: Bristol Conference report on defects in crystalline solids. The Physical Society of London, pp. 124-133.

Biscondi, M., Goux, C., 1968. Intercrystalline creep of oriented aluminium bi-crystals. Mem. Sci. Rev. Metall. 65, 167-179.

Bobylev, S. V., Mozorov, N. F., Ovid'ko, I. A., 2010. Cooperative grain boundary sliding and migration process in nanocrystalline solids. Phys. Rev. Lett. 105, 055504 . 
Bullough, R., 1965. The dislocation content of a large angle tilt boundary. Philos. Mag. 12, 1139-1141.

Bullough, R., Bilby, B. A., 1956. Continuous distributions of dislocations : surface dislocations and the crystallography of martensitic transformations. Proc. Phys. Soc. B 69, 1276-1286.

Busso, E. P., 1998. A continuum theory for dynamic recrystallization with microstructure-related length scales. Int. J. Plast. 14, 319-353.

Cahn, J. W., Mishin, Y., Suzuki, A., 2006a. Coupling grain boundary motion to shear deformation. Acta Mater. 54, 4953-4975.

Cahn, J. W., Mishin, Y., Suzuki, A., 2006b. Duality of dislocation content of grain boundaries. Philos. Mag. 86, 1-11.

Cahn, J. W., Taylor, J. E., 2004. A unified approach to motion of grain boundaries, relative tangential translation along grain boundaries and grain rotation. Acta Mater. 52, 4887-4898.

Cermelli, P., Gurtin, M. E., 1994. The dynamics of solid-solid phase transitions - 2. Incoherent interfaces. Arch. Rational. Mech. Anal. 127, 41-99.

Cherkaoui, M., Berveiller, M., Lemoine, X., 2000. Couplings between plasticity and martensitic phase transformation: overall behavior of polycrystalline TRIP steels. Int. J. Plast. 16, 1215-1241.

Cherkaoui, M., Berveiller, M., Sabar, H., 1998. Micromechanical modeling of martensitic transformation induced plasticity (TRIP) in austenitic single crystals. Int. J. Plast. 14, 597-626.

Chihab, K., Estrin, Y., Kubin, L. P., Vergnol, J., 1987. The kinetics of the Portevin-Le-Chatelier bands in an Al-5at.\% Mg alloy. Scripta Metall. 21, 203-208.

Christian, J. W., Mahajan, S., 1995. Deformation twinning. Prog. Mater. Sci. 39, $1-157$. 
Clayton, J. D., McDowell, D. L., Bammann, D. J., 2006. Modeling dislocations and disclinations with finite micropolar elastoplasticity. Int. J. Plast. 22, 210-256.

Coleman, B. D., Gurtin, M. E., 1967. Thermodynamics with internal state variables. J. Chem. Phys. 47, 597-613.

Dao, M., Lu, L., Asaro, R. J., De Hosson, J. T. M., Ma, E., 2007. Toward a quantitative understanding of mechanical behavior of nanocrystalline metals. Acta Materialia 55, 4041-4065.

Dederichs, P. H., Leibfried, G., 1969. Elastic Green's function for anisotropic cubic crystals. Phys. Rev. 188, 1175-1183.

Dvorak, G. J., 1990. On uniform fields in heterogeneous media. Proc. R. Soc. London A 431, 89-110.

Eshelby, J. D., 1951. The force on an elastic singularity. Phil. Trans. R. Soc. London A 244, 87-112.

Eshelby, J. D., 1970. Energy relations and energy-momentum tensor in continuum mechanics. In: Inelastic Behaviour of Solids. M. Kanninen et al. (Eds.),McGraw-Hill, New York, pp. 77-115.

Faken, D., Jonsson, H., 1994. Systematic analysis of local atomic structure combined with 3D computer graphics. Comput. Mater. Sci. 2, 279-286.

Farkas, D., Froseth, A., Swygenhoven, H. V., 2006. Grain boundary migration during room temperature deformation of nanocrystalline Ni. Scripta Mater. 55, 695-698.

Fischer, F. D., Schaden, T., Appel, F., Clemens, H., 2003. Mechanical twins, their development and growth. Eur. J. Mech. A. Solids 22, 709-726.

Fischer, F. D., Simha, N. K., Svoboda, J., 1998. Kinetics of diffusional transformation in multicomponent elastic-plastic materials. ASME J. Engng. Mater. Tech. 125, 266-276.

Franciosi, P., Berbenni, S., 2007. Heterogeneous crystal and polycrystal plasticity 
modeling from transformation field analysis within a regularized Schmid flow law. J. Mech. Phys. Solids 55, 2265-2299.

Franciosi, P., Berbenni, S., 2008. Multi-laminate plastic-strain organization for non-uniform TFA modeling of poly-crystal regularized plastic flow. Int. J. Plast. $24,1549-1580$.

Frank, F. C., 1950. The resultant content of dislocation in an arbitrary intercrystalline boundary. In: Symposium on the plastic deformation of crystalline solids. Carnegie Institute of Technology, Pittsburgh, pp. 150-154.

Gemperlova, J., Paidar, V., Kroupa, F., 1989. Compatibility stresses in deformed bicrystals. Czech. J. Phys. B 39, 427-446.

Gianola, D. S., Eberl, C., Cheng, X., Hemker, K. J., 2008. Stress-driven surface topography evolution in nanocrystalline Al thin films. Adv. Mater. 20, 303-308.

Gianola, D. S., Van Petegem, S., Legros, M., Brandstetter, S., Van Swygenhoven, H., Hemker, K. J., 2006. Stress-assisted discontinuous grain growth and its effect on the deformation behavior of nanocrystalline aluminum thin films. Acta Mater. 54, 2253-2263.

Gorkaya, T., Molodov, D. A., Gottstein, G., 2009. Stress-driven migration of symmetrical [100] tilt grain boundaries in Al bicrystals. Acta Mater. 57, $5396-5405$.

Gottstein, G., Shvindlerman, L., 2010. Grain boundary migration in metals (2nd ed.). CRC Press, Boca Raton, USA.

Hadamard, J., 1903. Lecons sur la propagation des ondes et les equations de l'hydrodynamique. College de France, Paris.

Hill, R., 1952. The elastic behaviour of a crystalline aggregate. Proc. Phys. Soc. Lond. 65, 349-354.

Hirth, J. P., Lothe, J., 1982. Theory of Dislocations (2nd ed.). Wiley, New York. Hirth, J. P., Pond, R. C., 1996. Steps, dislocations and disconnections as interface 
defects relating to structure and phase transformations. Acta Mater. 44, 4749-4763.

Hirth, J. P., Pond, R. C., Lothe, J., 2007. Spacing defects and disconnections in grain boundaries. Acta Mater. 55, 5428-5437.

Ivanov, V. A., Mishin, Y., 2008. Dynamics of grain boundary motion coupled to shear deformation: an analytical model and its verification by molecular dynamics. Phys. Rev. B 78, 064106.

Jin, M., Minor, A. M., Stach, E. A., Morris, J. W., 2004. Direct observation of deformation-induced grain growth during the nanoindentation of ultrafine-grained Al at room temperature. Acta Mater. 52, 5381-5387.

King, A. H., Smith, D. A., 1980. The effects on grain boundary processes of the steps in the boundary plane associated with the cores of grain boundary dislocations. Acta Crystal. A 36, 335-343.

Kröner, E., 1958. Kontinuumstheorie der Versetzungen und Eigenspannungen. Collatz L. and Loesch F. (eds.). Ergebnisse der Angewewandte Mathematik 5, Springer Verlag, Berlin.

Kröner, E., 1981. Continuum theory of defects. In: Physics of defects. R. Balian and al. (Eds.), Les Houches, Session 35, North Holland, New York, pp. 215-315. Kubin, L. P., Fressengeas, C., Ananthakrishna, G., 2002. Collective behaviour of dislocations in plasticity. In: Dislocations in Solids (vol. 11). Nabarro, F.R.N., Duesbery, M.S. (Eds.), Elsevier Science, Amsterdam, pp. 101-192.

Langlois, L., Berveiller, M., 2003. Overall softening and anisotropy related with the formation and evolution of dislocation cell structures. Int. J. Plast. 19, 599-624.

Legros, M., Gianola, D. S., Hemker, K. J., 2008. In situ TEM observations of fast grain-boundary motion in stressed nanocrystalline aluminium films. Acta Mater. $56,3380-3393$.

Li, C. H., Edwards, E. H., Washburn, J., Parker, E. R., 1953. Stress-induced 
movement of crystal boundaries. Acta Metall. 1, 223-229.

Li, J. C. M., 1961. High-angle tilt boundary - A dislocation core model. J. Appl. Phys. 32, 525-541.

Li, J. C. M., 1972. Disclination model of high angle grain boundaries. Surf. Sci. 31, $12-26$.

Luque, A., Aldazabal, J., Martinez-Esnaola, J. M., Gil Sevillano, J., 2010. Plastic deformation by conservative shear-coupled migration of tilt boundaries with intergranular nano-cracks or precipitates. Philos. Mag. 90, 3743-3756.

Maugin, G. A., 1992. The Thermomechanics of Plasticity and Fracture. Cambridge University Press.

Maugin, G. A., 2011. Configurational forces: Thermomechanics, Mathematics and Numerics. CRC Press, Taylor and Francis, New York.

Maugin, G. A., Muschik, W., 1994. Thermodynamics with internal variables: Part I. General concepts. J. Non-Equil. Thermodyn. 19, 217-249.

McDowell, D. L., 2008. Viscoplasticity of heterogeneous metallic materials. Mater. Sci. Eng. R 62, 67-123.

McDowell, D. L., 2010. A perspective on trends in multiscale plasticity. Int. J. Plast. 26, 1280-1309.

Mishin, Y., Mehl, M. J., Papaconstantopoulos, D. A., Voter, A. F., Kress, J. D., 2001. Structural stability and lattice defects in copper: Ab initio, tight-binding, and embedded-atom calculations. Phys. Rev. B 63, 224106.

Mishin, Y., Suzuki, A., Uberuaga, B. P., Voter, A. F., 2007. Stick-slip mechanism of grain boundaries studied by accelerated molecular dynamics. Phys. Rev. B 75, 224101.

Molodov, D. A., Gorkaya, T., Gottstein, G., 2007. Low angle tilt boundary migration coupled to shear deformation. Acta Mater. 55, 1843-1848.

Molodov, D. A., Gorkaya, T., Gottstein, G., 2011. Dynamics of grain boundaries 
under mechanical stress. J. Mater. Sci. 46, 4318-4326.

Mompiou, F., Caillard, D., Legros, M., 2009. Grain boundary shear-migration coupling: I. in situ TEM straining experiments in $\mathrm{Al}$ polycrystals. Acta Mater. $57,2198-2209$.

Mompiou, F., Caillard, D., Legros, M., 2010. SMIG model: a new geometrical model to quantify grain boundary-based plasticity. Acta Mater. 58, 3676-3689.

Mompiou, F., Caillard, D., Legros, M., 2011. Direct observation and quantification of grain boundary shear-migration coupling in polycrystalline Al. J. Mater. Sci. 46, 4308-4313.

Morawiec, A., 2009. On the frequency of occurrence of tilt and twist grain boundaries. Scripta Mater. 61, 438-440.

Mura, T., 1963. Continuous distribution of moving dislocations. Philos. Mag. 89, $843-857$.

Mura, T., 1987. Micromechanics of defects in solids. Kluwer Academic Publishers, Dordrecht, The Netherlands.

Nicaise, N., Berbenni, S., Wagner, F., Berveiller, M., Lemoine, X., 2011. Coupled effects of grain size distributions and crystallographic textures on the plastic behaviour of IF steels. Int. J. Plast. 27, 232-249.

Nye, J. F., 1953. Some geometrical relations in dislocated crystals. Acta Metall. 1, $153-162$.

Nye, J. F., 1957. Physical properties of crystals. Clarendon Press, Oxford.

Petryk, H., 1998. Macroscopic rate-variables in solids undergoing phase transformation. J. Mech. Phys. Solids 46, 873-894.

Pond, R. C., Ma, X., Hirth, J. P., 2008. Geometrical and physical models of martensitic transformations in ferrous alloys. J. Mater. Sci. 43, 3881-3888.

Rae, C. M. F., Smith, D. A., 1980. On the mechanisms of grain boundary migration. Philos. Mag. A 41, 477-492. 
Read, W. T., 1953. Dislocations in crystals. Mc Graw-Hill, New York.

Read, W. T., Shockley, W., 1950. Dislocation models of crystal grain boundaries. Phys. Rev. 78, 275-289.

Reuss, A., 1929. Calculation of the flow limits of mixed crystals on the basis of the plasticity of monocrystals. Z. Angew. Math. Mech. 9, 49-58.

Rey, C., Zaoui, A., 1980. Slip heterogeneities in deformed aluminium bicrystals. Acta Metall. 28, 687-697.

Rice, J. R., 1975. Continuum mechanics and thermodynamics of plasticity in relation to microscale deformation mechanisms. In: Constitutive equations in plasticity. A.S. Argon (ed.), MIT Press, Cambridge, pp. 23-79.

Richeton, T., Berbenni, S., 2013. Effects of heterogeneous elasticity coupled to plasticity on stresses and lattice rotations in bicrystals: a Field Dislocation Mechanics viewpoint. Eur. J. Mech. A. Solids 37, 231-247.

Schillebeeckx, C., Berbenni, S., Capolungo, L., Cherkaoui, M., 2011. A new micromechanics-based scale transition model for the strain-rate sensitive behavior of nanocrystalline materials. Philos. Mag. 91, 657-681.

Shiekhelsouk, M. N., Favier, V., Inal, K., Cherkaoui, M., 2009. Modelling the behaviour of polycrystalline austenitic steel with twinning-induced plasticity effect. Int. J. Plast. 25, 105-133.

Simha, N. K., Bhattacharya, K., 1998. Kinetics of phase boundaries with edges and junctions. J. Mech. Phys. Solids 46, 2323-2359.

Spearot, D. E., Capolungo, L., Qu, J., Cherkaoui, M., 2008. On the elastic tensile deformation of [001] bicrystal interfaces in copper. Comp. Mater. Sci. 42, 57-67.

Stupkiewicz, S., Petryk, H., 2002. Modelling of laminated microstructures in stress-induced martensitic transformations. J. Mech. Phys. Solids 50, 2303-2331. Sutton, A. P., Balluffi, R. W., 1995. Interfaces in Crystalline Solids. Clarendon Press, Oxford. 
Tsuzuki, H., Branicio, P. S., Rino, J. P., 2007. Structural characterization of deformed crystals by analysis of common atomic neighborhood. Comp. Phys. Comm. 177, 518-523.

Tucker, G. J., McDowell, D. L., 2011. Non-equilibrium grain boundary structure and inelastic deformation using atomistic simulations. Int. J. Plast. 27, 841-857.

Tucker, G. J., Zimmerman, J. A., McDowell, D. L., 2010. Shear deformation kinematics of bicrystalline grain boundaries in atomistic simulations. Modell. Simul. Mater. Sci. Eng. 18, 015002.

Tucker, G. J., Zimmerman, J. A., McDowell, D. L., 2011. Continuum metrics for deformation and microrotation from atomistic simulations: application to grain boundaries. Int. J. Eng. Sci. 49, 1424-1434.

Upadhyay, M., Capolungo, L., Taupin, V., Fressengeas, C., 2011. Grain boundary and triple junction energies in crystalline media: a disclination based approach. Int. J. Solids Struct. 48, 3176-3193.

Voigt, W., 1928. Lehrbuch der Kristallphysik. B. B. Teubner (ed.), Leipzig. Wang, J., Misra, A., Hirth, J. P., 2011. Shear response of twin boundaries in face-centered-cubic metals. Phys. Rev. B 83, 064106.

Warner, D. H., Sansoz, F., Molinari, J. F., 2006. Atomistic based continuum investigation of plastic deformation in nanocrystalline copper. Int. J. Plast. 22, $754-774$.

Willis, J. R., 1967. Second-order effects of dislocations in anisotropic crystals. Int. J. Eng. Sci. 5, 171-190.

Winning, M., Gottstein, G., Shvindlerman, L. S., 2001. Stress induced grain boundary motion. Acta Mater. 49, 211-219.

Winning, M., Gottstein, G., Shvindlerman, L. S., 2002. On the mechanisms of grain boundary migration. Acta Mater. 50, 353-363.

Wu, H. C., 2005. Continuum mechanics and plasticity (chapter 4). In: Modern 
Mechanics and Mathematics. D. Gao and R. W. Ogden (Eds.), Chapman-Hall, CRC Press, Boca Raton, USA, pp. 141-201.

Yoshida, H., Yokohama, K., Shibata, N., Ikuhara, Y., Sakuma, T., 2002.

High-temperature grain boundary sliding behaviour and grain boundary energy in cubic zirconia bicrystals. Acta Mater. 52, 2349-2357.

Zhang, H., Du, D., Srolovitz, D. J., 2008. Effects of boundary inclination and boundary type on shear-driven grain boundary migration. Philos. Mag. 88, $243-256$.

Zhang, K., Weertman, J. R., Eastman, J. A., 2005. Rapid stress-driven grain coarsening in nanocrystalline $\mathrm{Cu}$ at ambient and cryogenic temperatures. Appl. Phys. Lett. 87, 061921-3. 